\title{
Dynamical Friction and the Evolution of Supermassive Black Hole Binaries: The Final Hundred-parsec Problem
}

\author{
Fani Dosopoulou and Fabio Antonini \\ Center for Interdisciplinary Exploration and Research in Astrophysics (CIERA) and Department of Physics and Astrophysics, Northwestern University, Evanston, IL \\ 60208, USA; FaniDosopoulou2012@u.northwestern.edu, fabio.antonini@northwestern.edu \\ Received 2016 November 21; revised 2017 March 22; accepted 2017 April 2; published 2017 May 2
}

\begin{abstract}
The supermassive black holes originally in the nuclei of two merging galaxies will form a binary in the remnant core. The early evolution of the massive binary is driven by dynamical friction before the binary becomes "hard" and eventually reaches coalescence through gravitational-wave emission. We consider the dynamical friction evolution of massive binaries consisting of a secondary hole orbiting inside a stellar cusp dominated by a more massive central black hole. In our treatment, we include the frictional force from stars moving faster than the inspiralling object, which is neglected in the standard Chandrasekhar treatment. We show that the binary eccentricity increases if the stellar cusp density profile rises less steeply than $\rho \propto r^{-2}$. In cusps shallower than $\rho \propto r^{-1}$, the frictional timescale can become very long due to the deficit of stars moving slower than the massive body. Although including fast stars increases the decay rate, low mass-ratio binaries $\left(q \lesssim 10^{-3}\right)$ in sufficiently massive galaxies have decay timescales longer than one Hubble time. During such minor mergers, the secondary hole stalls on an eccentric orbit at a distance of order one-tenth the influence radius of the primary hole (i.e., $\approx 10-100 \mathrm{pc}$ for massive ellipticals). We calculate the expected number of stalled satellites as a function of the host galaxy mass and show that the brightest cluster galaxies should have $\gtrsim 1$ of such satellites orbiting within their cores. Our results could provide an explanation for a number of observations, which include multiple nuclei in core ellipticals, off-center AGNs, and eccentric nuclear disks.
\end{abstract}

Key words: galaxies: nuclei - stars: kinematics and dynamics - quasars: supermassive black holes

\section{Introduction}

A massive object moving through a cluster of lighter stars suffers a net deceleration along the direction of its motion known as dynamical friction. Dynamical friction can be understood as the drag induced on a test particle by the overdensity (i.e., the gravitational wake) that is raised behind it by the deflection of stars (Danby \& Camm 1957; Kalnajs 1972; Mulder 1983; Weinberg 1986). Dynamical friction is one of the most fundamental processes in astrophysics and its understanding is arguably the most important contribution of Chandrasekhar to stellar dynamics (Chandrasekhar 1943). Dynamical friction plays a key role in the evolution of supermassive black hole (SMBH) binaries (e.g., Merritt 2006), galaxies (e.g., van den Bosch et al. 1999), star clusters (e.g., Alessandrini et al. 2014), binary star cores in the common envelope phase of evolution (e.g., Paczynski 1976), and protoplanet migration (e.g., Ostriker 1999).

Chandrasekhar describes dynamical friction as the systematic decelerating effect of the fluctuating field of force acting on a star in motion. By assuming that the unperturbed motion of the test body was linear and unaccelerated, and that the fieldstar distribution was infinite and homogeneous spatially and isotropic in velocity space, Chandrasekhar derived an explicit formula for the dynamical friction force (Chandrasekhar 1943):

$$
\boldsymbol{F}_{\mathrm{df}}=-4 \pi G^{2} \frac{\boldsymbol{v}}{v^{3}}\left(m+m_{\star}\right) \ln \Lambda \int_{0}^{v} d v_{\star} 4 \pi f\left(v_{\star}\right) v_{\star}^{2},
$$

where $m_{\star}$ denotes the mass of the field stars, $m$ the mass of the test body, $v$ its velocity, $G$ the gravitational constant, $\ln \Lambda$ the Coulomb logarithm, and $f\left(v_{\star}\right)$ the stellar velocity distribution function. Clearly, Equation (1) implies that only stars with velocity $v_{\star}<v$, i.e., those that move slower than the test body, contribute to the decelerating force. Although Equation (1) has been shown to describe a variety of systems remarkably well (e.g., Spinnato et al. 2003; Baumgardt et al. 2006), deviations from the standard theory are also known to exist (e.g., Tremaine \& Weinberg 1984; Read et al. 2006; Gualandris \& Merritt 2008; Antonini \& Merritt 2012; Petts et al. 2016). The motivation for the work described in this paper is the existence of physically interesting models of galactic nuclei in which Equation (1) leads to erroneous results because the usual simplifying assumptions that lead to the contribution of stars with large velocities $\left(v_{\star}>v\right)$ to be neglected appear to break down.

In this paper, we present a comprehensive study of dynamical friction in the nuclei of galaxies containing an $\mathrm{SMBH}$. We derive the dynamical friction coefficients that describe the orbit-averaged time evolution of the energy and angular momentum of a test star near an SMBH. We do this by using the proper field-star velocity distribution (as opposed to, say, a Maxwellian), and including the contribution of the fast stars to the frictional force that was ignored in previous treatments of this problem. More specifically, analytical techniques and $N$-body simulations are used to test two predictions that the standard Chandrasekhar treatment makes about the evolution of a massive body moving near an SMBH. Assuming that the density of field stars follows $\rho(r) \sim r^{-\gamma}$, then Equation (1) predicts that (Antonini \& Merritt 2012) (i) the dynamical friction force goes to zero as $\gamma \rightarrow 1 / 2$ and (ii) the eccentricity is conserved for $\gamma=3 / 2$, while dynamical friction tends to circularize orbits for $\gamma>3 / 2$ and make them more eccentric for $\gamma<3 / 2$. Compared to previous work (e.g., Antonini \& Merritt 2012), we study the evolution of massive 
binaries in models with a wide range of density profiles and binary mass ratios and consider for the first time the effect of the friction from fast stars on the evolution of the binary eccentricity. Moreover, in our paper, the binary evolution equations are first derived using a perturbation approach based on the varying-conic method of Lagrange (e.g., Dosopoulou \& Kalogera 2016a) and then orbit-averaged (e.g., Dosopoulou \& Kalogera 2016b).

Predictions (i) and (ii) can be easily understood based on Equation (1), and noting that the distribution function that corresponds to an isotropic and spherical cluster of stars near an $\mathrm{SMBH}$ is $f(E) \propto|E|^{\gamma-3 / 2} / \Gamma(\gamma-1 / 2)$ (see also Equation (10) below), with $E$ the orbital energy. The previous expression shows that as $\gamma \rightarrow 1 / 2$, all stars have zero energy with respect to the $\mathrm{SMBH}$, i.e., the distribution function tends to a delta function that peaks at the local escape velocity. Under these conditions, Equation (1) implies that the dynamical friction force is zero, since there are no stars locally that move slower than the test star. Point (ii) is also easily shown to be true. For $\gamma=3 / 2, f(E)=$ const. Equation (1) in this case shows that the dynamical friction force is independent of radius and reduces to a linear deceleration $\operatorname{drag} \boldsymbol{F}_{\mathrm{df}}=-$ const $\cdot \boldsymbol{v}$ along the orbit. This implies that the orbital eccentricity of a massive body will remain unchanged during its motion. For steeper profiles, because the phase-space density is higher than average at periapsis, the additional drag there tends to circularize the orbit, while for shallower profiles the higher phase-space density at apoapsis tends to make the orbit more eccentric.

Our calculations show that the evolution of the test mass can be significantly affected by the frictional force produced by stars moving faster than its velocity. Adding this contribution leads to a timescale for inspiral that, for $1 / 2 \lesssim \gamma \lesssim 1$, can be up to one order of magnitude shorter than what is predicted by Equation (1). The orbital eccentricity of the test mass is found to increase during the inspiral for all values of $\gamma$ less than $\approx 2$; for steeper profiles, the eccentricity decreases but only mildly before the secondary SMBH reaches the center.

Finally, we consider the dynamical evolution of $\mathrm{SMBH}$ binaries, the formation of which is believed to be a generic product of galaxy mergers. We explore the dependence of the lifetime of an SMBH binary on its total mass, mass ratio, and on the density profile of the surrounding cusp. We calculate the expected number of stalled satellites as a function of the host galaxy SMBH mass and find that the inner cores of massive galaxies like M87 are likely hosts of stalled satellite SMBHs. The implications of our results are discussed in connection with a number of observations, which include off-center AGNs (Lena et al. 2014), binary AGNs (Rodriguez et al. 2006), double or multiple nuclei within core elliptical galaxies (Bonfini \& Graham 2016; Mazzalay et al. 2016), and eccentric nuclear disks (Lauer et al. 1996).

This paper is structured as follows. In Section 2 we introduce the general formalism we adopt to describe the orbital evolution of a massive binary moving inside a stellar cusp around a central $\mathrm{SMBH}$, treating dynamical friction as a perturbation to the classic Kepler problem. In Section 3, we compare the theoretical predictions for binary evolution with the results of $N$-body simulations. In Section 4 , we describe the different phases involved in the evolution of an SMBH binary and calculate the lifetime of an SMBH binary in early-type galaxies. In Section 5, we calculate the expected average number of stalled satellites in luminous galaxies as a function of the host galaxy SMBH mass, commenting on possible connections to observations. In Section 6, we present our conclusions.

\section{Analytical Treatment}

A binary system can be exposed to various perturbations emerging from physical processes involved in the course of its evolution. Within the astrophysical context, these processes are in principle dynamical processes in addition to the classic Newtonian gravity.

In stellar binaries, these processes include tidal forces and tidal friction, relativistic corrections, gravitational-wave emission, magnetic braking, mass-loss and mass-transfer interactions, as well as many-body forces. In massive binaries moving inside a stellar cusp, a fundamental perturbation is dynamical friction, which is the deceleration drag experienced by the secondary massive body.

Due to the various perturbations, each body in the binary is no longer moving in the actual Keplerian ellipse it would if no perturbations existed, but its physical orbit is slowly changing with time. The time evolution of the orbital elements can be described using the Varying-conic method advanced and completed by Lagrange. In this method, the true physical orbit of the body is approximated by a family of evolving instantaneous ellipses that at each moment in time describe the ellipse the body would follow if the perturbation ceased instantaneously.

In what follows, we describe the general formalism of this method, and we then apply this formalism to the orbital evolution of an inspiraling object inside a stellar cusp, treating dynamical friction as a perturbation to the binary orbit.

\subsection{Varying-conic Method}

The general reduced two-body problem where Newtonian gravity is the only force acting on the two bodies in the system is described by the equation

$$
\ddot{\boldsymbol{r}}=-\frac{G M}{r^{3}} \boldsymbol{r},
$$

where $G$ is the gravitational constant, $M$ is the total mass of the system, and $r$ is the relative position between the two bodies.

Any dynamical interaction between the two bodies introduces an extra force to the binary which acts as a perturbation to the classic two-body problem. Under the effect of a perturbing force $\boldsymbol{F}(\boldsymbol{r}, \dot{\boldsymbol{r}})$, the equation of motion for the perturbed two-body problem can be written as

$$
\ddot{\boldsymbol{r}}=-\frac{G M}{r^{3}} \boldsymbol{r}+\boldsymbol{F}(\boldsymbol{r}, \dot{\boldsymbol{r}}),
$$

where the perturbing force $\boldsymbol{F}(\boldsymbol{r}, \dot{\boldsymbol{r}})$ depends in principle upon both the relative position $\boldsymbol{r}$ and velocity $\boldsymbol{v}=\dot{\boldsymbol{r}}$.

Equation (3) can be solved assuming that at each instant of time, the true orbit can be approximated by an instantaneous ellipse that is changing over time through its now timedependent orbital elements $C_{i}(t)$. Here $C_{i}=(a, e, i, \Omega, \omega, f)$ are namely the semimajor axis, eccentricity, inclination, longitude of the ascending node, argument of periapsis, and true anomaly $f$. We also define $n=\left(G M / a^{3}\right)^{1 / 2}$ as the mean motion. At each moment of time, these orbital elements describe the orbit the body would follow if perturbations were 
to cease instantaneously. We refer to these elements as osculating orbital elements. The time-evolution equations for the osculating orbital elements decomposed in the reference system $K_{R}(\hat{\boldsymbol{r}}, \hat{\boldsymbol{\tau}}, \hat{\boldsymbol{n}})$, where the unit vector $\hat{\boldsymbol{r}}$ is along the relative position vector between the two bodies in the binary, become

$$
\begin{gathered}
\frac{d a}{d t}=\frac{2}{n \sqrt{1-e^{2}}}\left[F_{r} e \sin f+F_{\tau}(1+e \cos f)\right], \\
\frac{d e}{d t}=\frac{\sqrt{1-e^{2}}}{n a}\left[F_{r} \sin f+F_{\tau}\left(\cos f+\frac{e+\cos f}{1+e \cos f}\right)\right], \\
\frac{d \Omega}{d t}=\frac{d i}{n a^{2} \sqrt{1-e^{2}} \sin i} F_{n} \frac{a\left(1-e^{2}\right) \sin (f+\omega)}{1+e \cos f}, \\
\frac{d \omega}{d t}=\frac{\sqrt{1-e^{2}}}{n a e}\left(-F_{r} \cos f+F_{\tau} \sin f \frac{2+e \cos f}{1+e \cos f}\right) \\
-\frac{\cos i}{n a \sin i} F_{n} \frac{\sqrt{1-e^{2}} \sin (f+\omega)}{1+e \cos f}, \\
\frac{d f}{d t}=\frac{n(1+e \cos f)^{2}}{\left(1-e^{2}\right)^{3 / 2}}-\frac{d \omega}{d t}-\cos i \frac{d \Omega}{d t} .
\end{gathered}
$$

Equations (4) and (5) indicate that for a perturbing force always vertical to the orbital plane, i.e., $F_{r}=F_{\tau}=0$, the semimajor axis and the eccentricity do not change while Equations (6) and (7) show that the inclination $i$ and the longitude of the ascending node $\Omega$ evolve only for a non-zero vertical to the orbital plane component of the perturbing force, i.e., $F_{n} \neq 0$. On the contrary, from Equation (8) we see that the periapsis is precessing for any non-zero perturbing force, i.e., $\boldsymbol{F} \neq 0$.

In the following section, we apply the formalism described above to study the effect of dynamical friction on the orbital evolution of a test mass moving inside a cluster of stars around a central SMBH. In this case, dynamical friction acts as a perturbing force on the evolution of the inspiraling object. We begin with a brief introduction to dynamical friction as described initially by Chandrasekhar (1943) and further studied by Antonini \& Merritt (2012) in the case of a test mass moving inside a cluster of stars around a more massive central SMBH.

\subsection{Dynamical Friction}

In what follows we consider the evolution of a binary comprising a massive object moving near a SMBH of considerably larger mass and which sits at the center of a galaxy. Gravitational interaction with stars results in the loss of energy and angular momentum by the massive object. We assume that the galaxy is spherically symmetric and isotropic, and we describe it using a power-law stellar density profile of the form $\rho(r)=\rho_{0}\left(r / r_{0}\right)^{-\gamma}$, where $r_{0}$ is a characteristic radius and $\gamma$ is the slope of the density profile. In what follows, we set $r_{0}=r_{\text {infl }}$, with $r_{\text {infl }}$ the radius containing a mass in stars twice the mass of the central SMBH (M.), i.e., the SMBH influences radius. Unless otherwise specified, in what follows we use units such that $M_{\mathbf{0}}=r_{\text {infl }}=G=1$.

Assuming that the gravitational potential $\Phi$ is dominated by the central SMBH and neglecting the effect of the surrounding stars, we can write $\Phi \approx-G M . / r$. Eddington's formula then uniquely leads to the following distribution function of the field-star velocities (Merritt 2013, p. 544):

$$
f\left(v_{\star}\right)=\frac{\Gamma(\gamma+1)}{\Gamma\left(\gamma-\frac{1}{2}\right)} \frac{1}{2^{\gamma} \pi^{3 / 2} v_{\mathrm{c}}^{2 \gamma}}\left(2 v_{\mathrm{c}}^{2}-v_{\star}^{2}\right)^{\gamma-3 / 2},
$$

where $v_{\star}$ is the star velocity, $v_{\mathrm{c}}=\sqrt{G M \cdot / r}$ is the circular velocity, and the normalization corresponds to unit total number.

The general formula for the dynamical friction force also including the contribution from stars moving faster than the massive body is

$$
\begin{aligned}
\boldsymbol{F}_{\mathrm{df}} \approx & -4 \pi G^{2} m \rho(r) \frac{\boldsymbol{v}}{v^{3}} \times\left\{\ln \Lambda \int_{0}^{v} d v_{\star} 4 \pi f\left(v_{\star}\right) v_{\star}^{2}\right. \\
& \left.+\int_{v}^{v_{e s c}} d v_{\star} 4 \pi f\left(v_{\star}\right) v_{\star}^{2}\left[\ln \left(\frac{v_{\star}+v}{v_{\star}-v}\right)-2 \frac{v}{v_{\star}}\right]\right\},
\end{aligned}
$$

where $\boldsymbol{v}$ is the velocity of the massive body and $m$ its mass. The quantity $\ln \Lambda$ is the Coulomb logarithm defined as

$$
\ln \Lambda=\ln \left(\frac{b_{\max }}{b_{\min }}\right) \approx \ln \left(\frac{b_{\max } v_{\mathrm{c}}^{2}}{G m}\right),
$$

where $b_{\max }$ and $b_{\min }$ are the maximum and minimum impact parameters, respectively.

We can rewrite the dynamical friction force in a more compact form as

$$
\begin{gathered}
\boldsymbol{F}_{\mathrm{df}}=\kappa(r)[\ln \Lambda \alpha(v)+\beta(v)+\delta(v)] \frac{\boldsymbol{v}}{v^{3}}, \\
\boldsymbol{F}_{\mathrm{df}}=\epsilon(r, v) \frac{\boldsymbol{v}}{v^{3}},
\end{gathered}
$$

where we defined

$$
\begin{gathered}
\kappa(r)=-4 \pi G^{2} \rho(r) m, \\
\epsilon(r, v)=\kappa(r)[\ln \Lambda \alpha(v)+\beta(v)+\delta(v)], \\
\alpha(v)=4 \pi \int_{0}^{v} f\left(v_{\star}\right) v_{\star}^{2} d v_{\star}, \\
\beta(v)=4 \pi \int_{v}^{v_{\mathrm{esc}}} f\left(v_{\star}\right) v_{\star}^{2}\left[\ln \left(\frac{v_{\star}+v}{v_{\star}-v}\right)\right] d v_{\star}, \\
\delta(v)=4 \pi v \int_{v}^{v_{\mathrm{esc}}} f\left(v_{\star}\right)\left(-2 v_{\star}\right) d v_{\star} .
\end{gathered}
$$

The distribution function (10) can be rewritten as

$$
f\left(v_{\star}\right)=\frac{\Gamma(\gamma+1)}{\Gamma\left(\gamma-\frac{1}{2}\right)} \frac{1}{2^{\gamma} \pi^{3 / 2} v_{\mathrm{c}}^{3}}\left[2-x^{2}\right]^{b}
$$

where we defined $x=v_{\star} / v_{\mathrm{c}}$ and $b=\gamma-3 / 2$. Integrals (17) and (19) have an analytic form while integral (18) demands numerical manipulation. Using Equation (20), we can rewrite the above integrals in dimensionless form as

$$
\begin{array}{r}
\alpha(\xi)=\frac{\Gamma(\gamma+1)}{\Gamma\left(\gamma-\frac{1}{2}\right)} \frac{4}{3} \pi^{-1 / 2} 2^{b-\gamma} \xi^{3} \\
\times{ }_{2} F_{1}\left[3 / 2,-b, 5 / 2, \xi^{2} / 2\right], \\
\beta(\xi)=\frac{\Gamma(\gamma+1)}{\Gamma\left(\gamma-\frac{1}{2}\right)} 4 \pi^{-1 / 2} 2^{-\gamma},
\end{array}
$$




$$
\begin{gathered}
\times \int_{\xi}^{1.4} x^{2}\left(2-x^{2}\right)^{b} \ln \left(\frac{x+\xi}{x-\xi}\right) d x \\
\delta(\xi)=\frac{\Gamma(\gamma+1)}{\Gamma\left(\gamma-\frac{1}{2}\right)} 8 \pi^{-1 / 2} \frac{2^{-\gamma-1}}{b+1} \xi \\
\times\left[0.04^{b+1}-\left(2-\xi^{2}\right)^{b+1}\right]
\end{gathered}
$$

where $\xi=v / v_{c}$, and we made use of that fact that $v_{\mathrm{esc}} / v_{\mathrm{c}}=\sqrt{2}$.

The orbital velocity and dynamical friction force (14) decomposed in the reference system $K_{R}(\hat{\boldsymbol{r}}, \hat{\boldsymbol{\tau}}, \hat{\boldsymbol{n}})$ mentioned above can be written as

$$
\begin{gathered}
\boldsymbol{v}=v_{r} \hat{\boldsymbol{r}}+v_{\tau} \hat{\boldsymbol{\tau}}+v_{n} \hat{z}, \\
\boldsymbol{F}_{\mathrm{df}}=F_{\mathrm{df}, r} \hat{\boldsymbol{r}}+F_{\mathrm{df}, \tau} \hat{\boldsymbol{\tau}}+F_{\mathrm{df}, n} \hat{z} .
\end{gathered}
$$

Substituting Equation (26) into Equation (14), we have $F_{\mathrm{df}, r}=\epsilon(r, v) \frac{v_{r}}{v^{3}}, F_{\mathrm{df}, \tau}=\epsilon(r, v) \frac{v_{\tau}}{v^{3}}$ and $F_{\mathrm{df}, n}=v_{n}=0$.

Using the dynamical friction components derived above and Equations (4)-(9), the osculating orbital element time-evolution equations of an inspiraling massive body due to dynamical friction are

$$
\begin{gathered}
\frac{d a}{d t}=\frac{2 \epsilon(r, v)}{n^{3} a^{2}} \frac{\left(1-e^{2}\right)^{1 / 2}}{\left(1+e^{2}+2 e \cos f\right)^{1 / 2}} \\
\frac{d e}{d t}=\frac{2 \epsilon(r, v)}{n^{3} a^{3}}\left(1-e^{2}\right)^{3 / 2} \frac{e+\cos f}{\left(1+e^{2}+2 e \cos f\right)^{3 / 2}} \\
\frac{d i}{d t}=F_{\mathrm{df}, n} \frac{\cos (f+\omega) \sqrt{1-e^{2}}}{n a(1+e \cos f)}=0 \\
\frac{1}{n a^{2} \sqrt{1-e^{2}} \sin i} F_{\mathrm{df}, n} \frac{\cos (f+\omega) \sqrt{1-e^{2}}}{n a(1+e \cos f)}=0 \\
\frac{d \omega}{d t}=\frac{2 \epsilon(r, v)}{n^{3} a^{3}} \frac{\left(1-e^{2}\right)^{3 / 2}}{\left(1+e^{2}+2 e \cos f\right)^{3 / 2}} \frac{\sin f}{e} \\
\frac{d f}{d t}=\frac{n(1+e \cos f)^{2}}{\left(1-e^{2}\right)^{3 / 2}}-\frac{d \omega}{d t}
\end{gathered}
$$

Equations (28)-(33) verify the aforementioned comment that in the absence of a vertical to the orbital plane component of the perturbing force, which is true in the case of dynamical friction $\left(F_{\mathrm{df}, n}=0\right)$, the inclination $i$ and the longitude of the ascending node $\Omega$ remain constant in the absence of other perturbing forces. Although according to Equation (32) dynamical friction can in principle induce a precession to the orbit, this precession has a negligible effect on the evolution of $e$ and $a$.

\subsection{Eccentricity Evolution}

We focus here on how dynamical friction affects the binary eccentricity. We begin by investigating qualitatively the expected eccentricity evolution using a simplified physical picture of the problem. This picture focuses on the eccentricity changes near periapsis and apoapsis. This analysis is useful to understand the link between the expected eccentricity evolution of the system and the physical origin of the dynamical friction force. The time evolution of the eccentricity vector $\boldsymbol{e}$ induced by a perturbing force $\boldsymbol{F}$ (in our case $\boldsymbol{F}=\boldsymbol{F}_{\mathrm{df}}$ ) is given by

$$
\dot{\boldsymbol{e}}=\frac{1}{G M}\left(\boldsymbol{F}_{\mathrm{df}} \times \boldsymbol{h}+\boldsymbol{v} \times \dot{\boldsymbol{h}}\right),
$$

where $\boldsymbol{h}=\boldsymbol{r} \times \boldsymbol{v}$ is the angular momentum per unit reduced mass $\mu=M \cdot m /(M \cdot+m)$, and the dot indicates a derivative with respect to time. In the absence of other perturbing forces in the binary, the angular momentum changes are only due to dynamical friction.

The dynamical friction force given by Equation (11) is a decelerating drag force (i.e., $\boldsymbol{F}_{\mathrm{df}}=-g(v, r) \boldsymbol{v}$, where $g(v, r)$ is a function of the massive body position and velocity) always acting in the direction opposite to the direction of motion of the body. In addition, the time evolution of the angular momentum vector is given by $\dot{\boldsymbol{h}}=\boldsymbol{r} \times \boldsymbol{F}_{\mathrm{df}} \Rightarrow \dot{\boldsymbol{h}}=-g(v, r) \boldsymbol{r} \times \boldsymbol{v}$. This leads to $\dot{\boldsymbol{e}}=-2 g(v, r)\left[v^{2} \boldsymbol{r}-(\boldsymbol{v} \cdot \boldsymbol{r}) \boldsymbol{v}\right]$. The eccentricity vector $\boldsymbol{e}$ is defined as always pointing in the direction of periapsis. This indicates that the eccentricity rate induced at periapsis is $\dot{e}_{\mathrm{p}}=\dot{\boldsymbol{e}} \cdot \hat{\boldsymbol{r}}<0$, tending to decrease the eccentricity and circularize the orbit, while at apoapsis $\dot{e}_{\mathrm{a}}=\dot{\boldsymbol{e}} \cdot \hat{\boldsymbol{r}}>0$, tending to increase the eccentricity (note that $\boldsymbol{v} \cdot \boldsymbol{r}=0$ at both periapsis and apoapsis). In addition, Equation (34) shows that for a massive body in an elliptical orbit, the eccentricity decrease rate due to dynamical friction is maximized at periapsis $(f=0)$ while the eccentricity increase rate is maximized at apoapsis $(f=\pi)$. This implies that the eccentricity evolution depends on the relative time the massive body spends near periapsis and apoapsis along the orbit. Using this simplified picture, we calculate below the expected eccentricity evolution of the binary orbit.

Equation (29) gives the instantaneous change of the eccentricity

$$
\begin{aligned}
& \left(\frac{d e}{d t}\right)_{\mathrm{p}}=\frac{2\left(1-e^{2}\right)^{3 / 2}}{n^{3} a^{3}} \frac{\epsilon_{\mathrm{p}}}{(1+e)^{2}}, \\
& \left(\frac{d e}{d t}\right)_{\mathrm{a}}=-\frac{2\left(1-e^{2}\right)^{3 / 2}}{n^{3} a^{3}} \frac{\epsilon_{\mathrm{a}}}{(1-e)^{2}},
\end{aligned}
$$

with $\epsilon_{\mathrm{p}}$ and $\epsilon_{\mathrm{a}}$ the value of $\epsilon(r, v)$ calculated at periapsis and apoapsis, respectively. The time spent by the massive body near periapsis $\left(\Delta \tau_{\mathrm{p}}\right)$ or apoapsis $\left(\Delta \tau_{\mathrm{a}}\right)$ is proportional to

$$
\begin{aligned}
& \Delta \tau_{\mathrm{p}} \propto \frac{1}{v_{\mathrm{p}}} \sim \sqrt{\frac{1-e}{1+e}}, \\
& \Delta \tau_{\mathrm{a}} \propto \frac{1}{v_{\mathrm{a}}} \sim \sqrt{\frac{1+e}{1-e}} .
\end{aligned}
$$

The expected increase or decrease of the eccentricity can then be determined by the ratio

$$
\zeta \equiv \frac{\Delta e_{\mathrm{p}}}{\Delta e_{\mathrm{a}}}=\frac{(d e / d t)_{\mathrm{p}} \Delta \tau_{\mathrm{p}}}{(d e / d t)_{\mathrm{a}} \Delta \tau_{\mathrm{a}}},
$$

where $\Delta e_{\mathrm{p}}$ and $\Delta e_{\mathrm{a}}$ are the induced eccentricity changes near periapsis and apoapsis, respectively. If $|\zeta|=1$ the eccentricity remains constant, if $|\zeta|>1$ the contribution near periapsis dominates and the eccentricity decreases, while if $|\zeta|<1$ the apoapsis contribution dominates and the eccentricity increases.

We note that Equations (35) and (36) reduce to analytic expressions if we take into account only stars moving slower than the massive body. Under this consideration, we have 


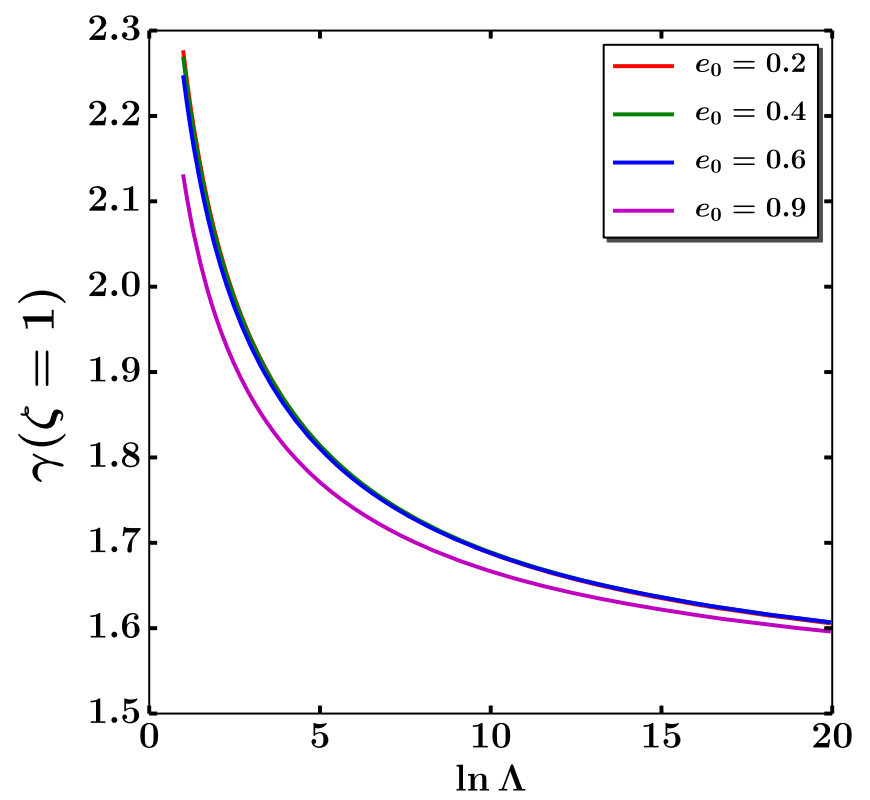

Figure 1. Value of $\gamma$ for which $\zeta=1$ as a function of the Coulomb logarithm $\ln \Lambda$, and for different initial eccentricities $e_{0}$. The eccentricity of the binary orbit increases with time for any $\gamma<\gamma(\zeta=1)$. Note that if only the slow stars are included, $\gamma(\zeta=1)=1.5$ for any value of $\ln \Lambda$ and $e_{0}$.

$$
\begin{gathered}
\beta(\xi)=\gamma(\xi)=0 \text { and Equations (16) and (17) lead to } \\
\qquad \epsilon_{\mathrm{p}} \propto(1-e)^{-\gamma}(1+e)^{3 / 2}{ }_{2} F_{1}^{p}, \\
\epsilon_{\mathrm{a}} \propto(1+e)^{-\gamma}(1-e)^{3 / 2}{ }_{2} F_{1}^{a} .
\end{gathered}
$$

Substituting Equations (40) and (41) into Equations (35) and (36), we have

$$
\begin{gathered}
\left(\frac{d e}{d t}\right)_{\mathrm{p}} \propto-\frac{(1-e)^{-\gamma}}{(1+e)^{1 / 2}}{ }_{2} F_{1}^{p}, \\
\left(\frac{d e}{d t}\right)_{\mathrm{a}} \propto \frac{(1+e)^{-\gamma}}{(1-e)^{1 / 2}} \quad{ }_{2} F_{1}^{a},
\end{gathered}
$$

where the hypergeometric function ${ }_{2} F_{1}$ is always positive for all $0<e<1$. The different signs in Equations (42) and (43) confirm the fact that dynamical friction tends to circularize the orbit at periapsis $\left(\dot{e}_{\mathrm{p}}<0\right)$ while at apoapsis tends to increase it $\left(\dot{e}_{\mathrm{a}}>0\right.$ ). Combining Equations (37), (38), (42), and (43), the ratio (39) is simplified to

$$
|\zeta|=\left(\frac{1-e}{1+e}\right)^{3 / 2-\gamma} \frac{{ }_{2} F_{1}^{p}}{{ }_{2} F_{1}^{a}} .
$$

As expected, for $\gamma=3 / 2$, we find $|\zeta|=1$ and the eccentricity remains constant; for $\gamma>3 / 2$, the first term on the right-hand side of Equation (44) is $>1$ and ${ }_{2} F_{1}^{p}>{ }_{2} F_{1}^{a}$. In this case, Equation (44) leads to $|\zeta|>1$. On the other hand, for $\gamma<3 / 2$ we have ${ }_{2} F_{1}^{p}<{ }_{2} F_{1}^{a}$ and $|\zeta|<1$.

Figure 1 demonstrates the expected eccentricity evolution. When the contribution of fast stars is included, the critical value of $\gamma$ below which the eccentricity increases is no longer $3 / 2$ but $\approx 2$. This critical value of $\gamma$ is found to depend slightly on the initial eccentricity while a smaller $\ln \Lambda$ increases the parameter space for which the binary eccentricity increases. The fact that the critical value of $\gamma$ in this case is greater than $3 / 2$ reflects the fact that the relative contribution of the fast stars is larger near apoapsis where the massive body is moving slower than the local circular velocity. This results in an enhanced drag force at apoapsis and higher eccentricities.

\subsection{Secular Evolution}

The orbital element time-evolution Equations (28)-(33) of a perturbed binary are in principle phase dependent and undergo physical oscillations with the orbital period. For a perturbation with a long timescale compared to the orbital period, these oscillations can be smoothed out by adopting orbit-averaging techniques. The orbit-averaged orbital element time-evolution equations then describe the secular evolution of the system.

In order to orbit-average a quantity along the orbit, we need to know how the true anomaly is changing over time. This is described by Equation (9), which shows that apart from the unperturbed Keplerian evolution described by the first term on the right-hand side of this equation, the true anomaly can also evolve due to possible precessions and specifically the periapsis precession $\dot{\omega}$ and the longitude of the ascending node precession $\Omega$. Given that due to dynamical friction we have $\dot{\omega} \ll 1$ and that $\dot{\Omega}=0$, we can compute the secular evolution of the orbital elements neglecting the second term in Equation (9) and use

$$
d f=n \frac{(1+e \cos f)^{2}}{\left(1-e^{2}\right)^{3 / 2}} d t
$$

when integrating Equations (28) and (29) over the orbit. In order to incorporate higher-order effects in the time-evolution equations, we have to proceed one step further and include all the terms in Equation (9) when applying the orbit-averaging process. In this paper, we derive first-order secular timeevolution equations where the general orbit-averaging rule for the phase-dependent quantity (...) is defined by the integral

$$
\langle(\ldots)\rangle=\frac{\left(1-e^{2}\right)^{3 / 2}}{2 \pi} \int_{0}^{2 \pi} \text { (..) } \frac{d f}{(1+e \cos f)^{2}} .
$$

Under these considerations, the secular time-evolution equations for the semimajor axis and the eccentricity of the massive body can be written as

$$
\begin{aligned}
\left\langle\frac{d a}{d t}\right\rangle & =\frac{2\left(1-e^{2}\right)^{2}}{\pi n^{3} a^{2}} \int_{0}^{2 \pi} \frac{(1+e \cos f)^{-2} \epsilon(r, v)}{\left(1+e^{2}+2 e \cos f\right)^{1 / 2}} d f, \\
\left\langle\frac{d e}{d t}\right\rangle= & \frac{2\left(1-e^{2}\right)^{3}}{\pi n^{3} a^{3}} \\
& \times \int_{0}^{2 \pi} \frac{(e+\cos f) \epsilon(r, v)}{\left(1+e^{2}+2 e \cos f\right)^{3 / 2}(1+e \cos f)^{2}} d f .
\end{aligned}
$$

Making use of Equation (47), we plot in Figure 2 the secular semimajor axis evolution of an inspiraling body with mass ratio $q=m / M_{0}=10^{-3}$. We see that for $\gamma<1$ the dynamical friction timescale becomes much longer, although the contribution to dynamical fiction from the fast stars always increases the orbital decay rate. We discuss the implications of the long dynamical friction timescale for SMBH binaries in early-type galaxies in Sections 4 and 5 below.

The secular evolution of the binary eccentricity is described by Equation (48). Using this equation we plot in Figure 3 the average fractional change in the quantity $R=1-e^{2}$ in one 


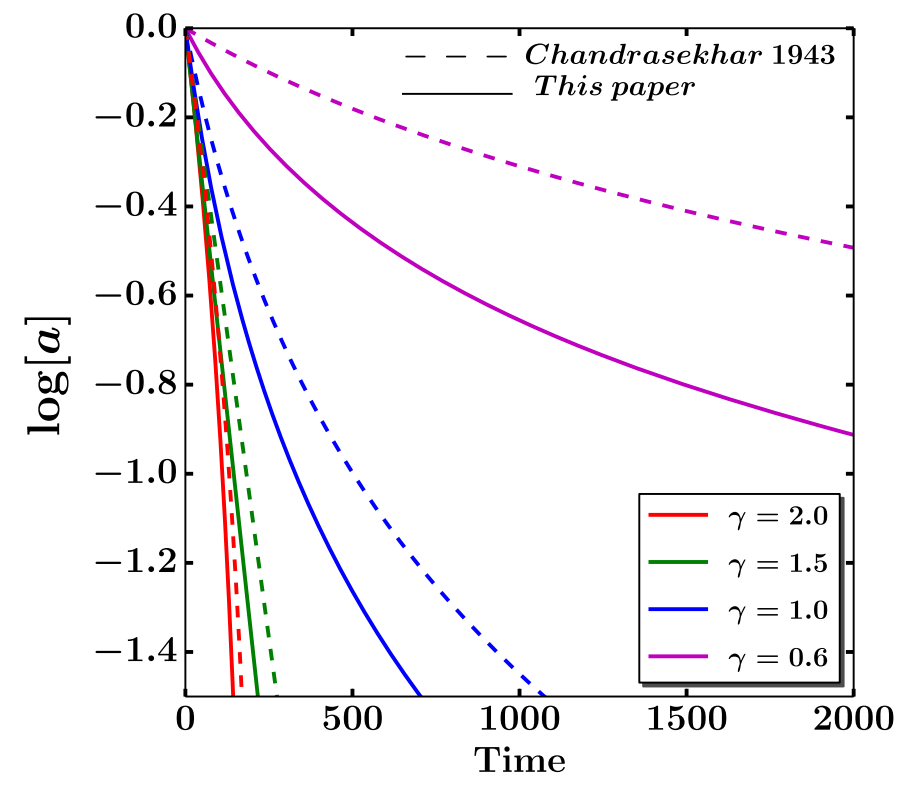

Figure 2. Secular evolution of the semimajor axis for a massive binary with $q=10^{-3}$ and for different slopes $\gamma$ of the density profile. Dashed lines include only the contribution of slow stars to dynamical friction (Chandrasekhar 1943), while solid lines also include the contribution from fast stars. In shallow stellar cusps with $\gamma<1$, the dynamical friction timescale becomes long and the orbital decay is slow. Adding the contribution from the fast stars increases the orbital decay rate. In this calculation we set $\ln \Lambda=6.0$ and $e_{0}=0.1$.

orbital decay time, i.e., $|a / \dot{a}|$. We plot this change as a function of the initial eccentricity.

In the upper panel of Figure 3, we see that adding the contribution of fast stars makes the eccentricity higher compared to the case where only the slow stars were taken into account. The results shown in Figure 3 clearly imply that the change in eccentricity can be of order unity if $\gamma<1$.

In the lower panel of Figure 3, we compare the results of Equation (48) with the eccentricity change predicted assuming that the stellar velocities follow a Maxwellian distribution. As expected, the use of a Maxwellian velocity distribution is inadequate to describe the evolution of the binary. More specifically, adopting a Maxwellian distribution always leads to a shorter timescale for the eccentricity evolution compared to what we obtain by using the distribution function (10).

\section{3. $N$-body Treatment}

Chandrasekhar formulated his theory assuming an infinite and homogeneous field of stars and that the unperturbed fieldstar trajectories are straight lines. Any of these assumptions represents a simplification of the real physical system; both the test and field particles for example move on ellipses, not straight lines, around the central SMBH. Thus, it is not clear whether Equations (47) and (48) can accurately describe the dynamical evolution of the massive binary due to dynamical friction. Antonini \& Merritt (2012) showed that Chandrasekhar's theory reproduces remarkably well the real decay rate of a massive object into a shallow density profile model; however, this is only when the contribution of the fast stars is included in evaluating the frictional drag. Whether the theory also reproduces the evolution of the binary eccentricity as well as the orbital decay for a range of density profile slopes remains to be shown. Here we use $N$-body simulations to investigate the evolution of a massive binary starting with various initial eccentricities and density profile models.

In order to validate Chandrasekhar's treatment in the systems we considered, the results of the simulations are compared to theoretical predictions based on Equations (47) and (48). In order to make such a comparison, in this section we adopt $\ln \Lambda \approx \ln (M . / m)$, which gives values consistent with those found in previous studies (Spinnato et al. 2003; Antonini \& Merritt 2012) and can be derived analytically from Equation (12) if one identifies $b_{\max }$ with the local scale length determined by the density gradient $\rho /|\nabla \rho|$ (Just et al. 2011).

Before we discuss the results of our $N$-body integrations, we introduce here two critical values of the binary separation which will turn out to be crucial for the correct interpretation of our models. Dynamical friction is expected to only affect the evolution of the massive binary until its separation reaches the semimajor axis of a "hard" binary, ${ }^{1}$ which is often expressed as (Merritt 2013)

$$
a_{\mathrm{h}} \approx 36 \frac{q}{(1+q)^{2}} \frac{M \cdot+m}{3 \times 10^{9} M_{\odot}}\left(\frac{\sigma}{300 \mathrm{~km} \mathrm{~s}^{-1}}\right)^{-2} \mathrm{pc},
$$

with (Alexander 2005)

$$
\sigma^{2}=\frac{1}{1+\gamma} \frac{G M}{r}
$$

the stellar velocity dispersion of the primary galaxy. At $a_{\mathrm{h}}$ the evolution of the massive binary ceases to be driven by dynamical friction, and its semimajor axis shrinks as the two massive objects interact with stars and eject them from the nucleus via gravitational slingshots. Even before the binary reaches $a_{\mathrm{h}}$, our analytical treatment is expected to break down as the binary separation becomes smaller than the radius containing a mass equal to the mass of the inspiraling body

$$
a_{\text {crit }} \equiv r\left(M_{\mathrm{m}}=m\right)=r_{\text {infl }}\left(\frac{m}{2 M \bullet}\right)^{\frac{1}{3-\gamma}},
$$

with $M_{\mathrm{m}}(r)$ the mass in stars within a sphere of radius $r$ from the primary SMBH. At $a \lesssim a_{\text {crit }}$ the analytical treatment breaks down as the star distribution in the cluster starts to be significantly affected by the motion of the massive intruder (e.g., Baumgardt et al. 2006; Matsubayashi et al. 2007).

\subsection{Initial Setup and Numerical Method}

We generate equilibrium $N$-body models of stars around an $\mathrm{SMBH}$ by adopting the truncated mass model

$$
\rho(r)=\rho_{0}\left(\frac{r}{r_{\text {infl }}}\right)^{-\gamma} \exp \left(-r / r_{\text {tr }}\right) .
$$

Monte Carlo initial positions and velocities were assigned to the $N$-body particles by numerically generating the distribution function corresponding to the isotropic equilibrium model of Equation (52), while at the same time taking into account the gravitational potential due to the central SMBH. A massive particle was placed in these models with mass $m \gg m_{\star}$, with corresponding mass ratio $q \ll 1$ at an initial galactocentric distance $r \lesssim r_{\text {tr }}$.

\footnotetext{
1 Defined as a binary that ejects passing stars at typical velocities greater than the escape velocity from the nucleus.
} 

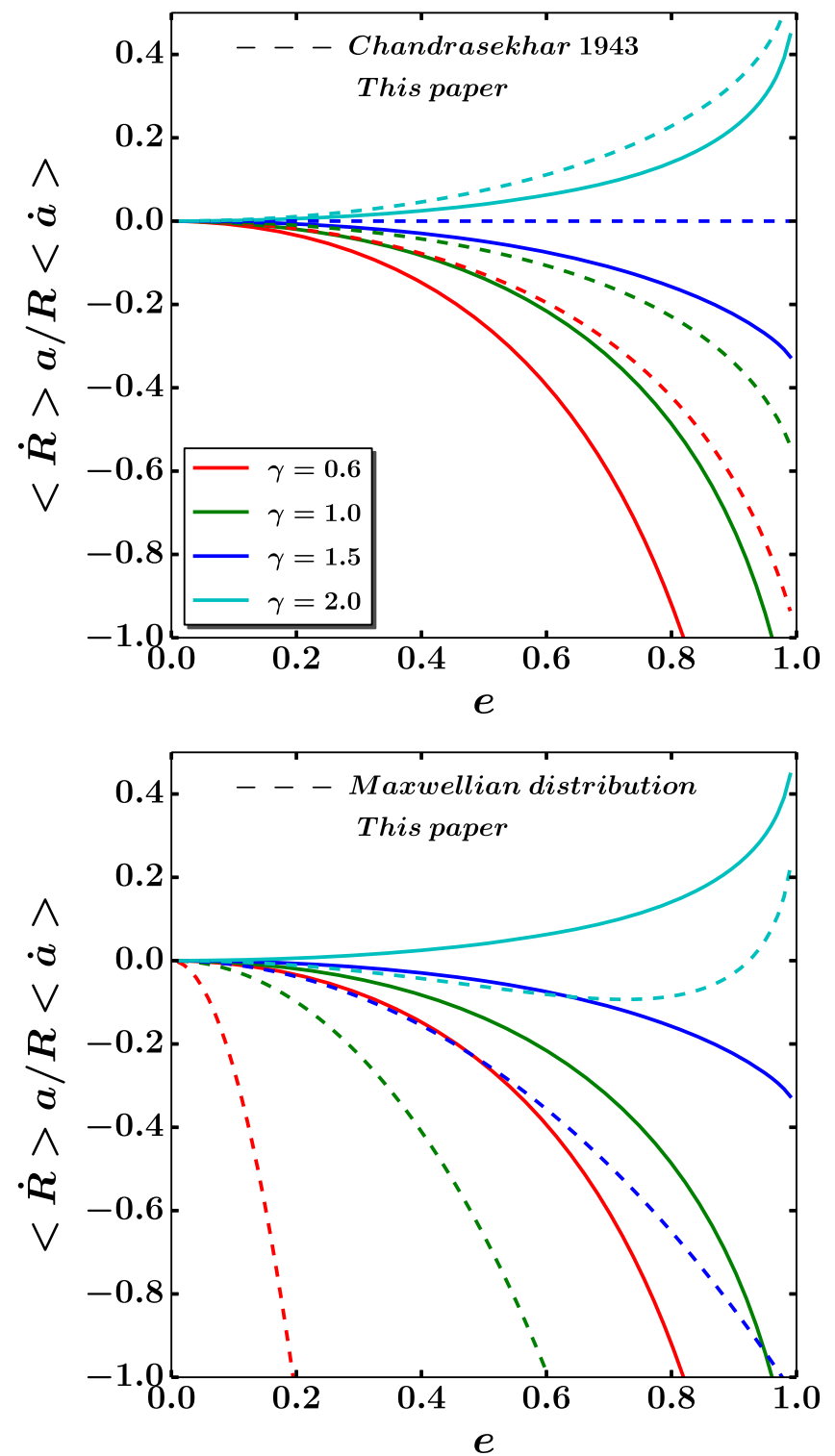

Figure 3. Average fractional change in the quantity $R=1-e^{2}$ by the time the massive body reaches the center as a function of the initial eccentricity. Solid lines include the contribution from the fast stars. The dashed lines in the upper panel include only the contribution from stars moving slower than the test mass (e.g., Figure 8.18 in Merritt 2013), while in the lower panel they are obtained by using a Maxwellian stellar velocity distribution. The eccentricity always increases unless $\gamma \sim 2$, and it is always higher when including the fast stars. Assuming a Maxwellian velocity distribution appears to be a poor approximation for any value of $\gamma$.

The initial conditions were evolved forward in time using the direct-summation code $\phi$ GRAPE (Harfst et al. 2007). This code uses a fourth-order Hermite integrator with a predictorcorrector scheme and hierarchical time steps. The performance and accuracy of the code depend both on the time-step parameter $\eta$ and on the smoothing length $\epsilon$. We set $\eta=0.01$ and $\epsilon=5 \times 10^{-4}$. With these choices, energy conservation was typically of order $0.1 \%$ over the entire length of the integration. The calculations were carried out in serial mode using graphics processing units combined with the SAPPORO library (Gaburov et al. 2009; Bédorf et al. 2015).

\subsection{Results}

Figure 4 shows the evolution of the semimajor axis and eccentricity of the massive binary in $N$-body models with $\gamma=(0.6,1.5,2)$, two values of binary mass ratio $q=$ $\left(2 \times 10^{-4}, 5 \times 10^{-5}\right)$, and two different values for the mass of the field particles $m_{\star}=\left(5 \times 10^{-6}, 1 \times 10^{-5}\right)$. In all the models shown in Figure 4, we set $r_{\text {tr }}=0.2$ and place the secondary massive body at $r=r_{\text {tr }}$ with a velocity half the circular value. With this choice of parameters, the initial values of semimajor axis and eccentricity are $a_{0} \approx 0.1$ and $e_{0} \approx 0.7$.

The resulting eccentricity and semimajor axis evolution in the simulations shown in Figure 4 are in good agreement with the theoretical predictions. In all cases, the semimajor axis evolves more rapidly than the eccentricity does. Indeed, we find no systematic change in the binary eccentricity over the simulated timescale for any value of $\gamma$. Although this behavior seems largely consistent with the predicted evolution, we also observe random-like variations of the orbital eccentricity. Such fluctuations in $e$ are due to the hard scattering off surrounding stars which causes the angular momentum of the massive body to random walk with an amplitude that decreases with increasing mass ratio $m / m_{\star}$ (e.g., Matsubayashi et al. 2007). We note that in a real galaxy the mass ratio between the 

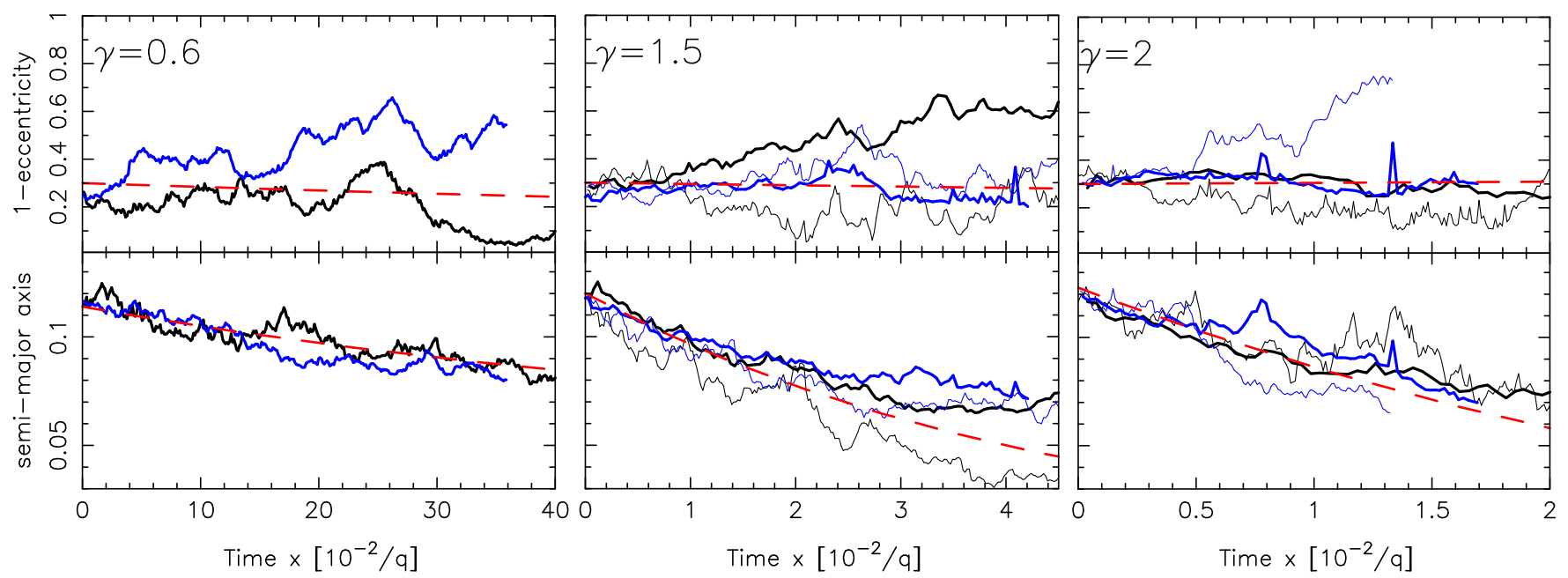

Figure 4. Evolution of the semimajor axis and eccentricity of the massive binary in the $N$-body simulations. Results for $m_{\star}=\left(5 \times 10^{-6}\right.$ and $\left.1 \times 10^{-5}\right)$ are given by the black and blue lines, respectively. Line thickness increases with the mass ratio of the binary for which we considered the two values $q=\left(2 \times 10^{-4} ; 5 \times 10^{-5}\right)$. Simulations shown are in good agreement with the theoretical prediction (dashed red lines), which was obtained from Equation $(48)$ with $\ln \Lambda \approx \ln \left(1 / 10^{-4}\right)$.

secondary massive body and field stars could be much larger than in our simulations, so such an effect would be essentially absent.

Figures 5 and 6 show simulations where the orbit of the massive body was followed until $a$ had shrunk by a factor of $\approx 100$ below its initial value. In these additional simulations, we increased the mass of the secondary body and field particles, and set $r_{\text {tr }}=1$.

In the simulations shown in Figure $5, a_{0} \approx 1$ and $e_{0} \approx 0.3$. We see from the left panels that the orbital evolution of the binary at $a \gtrsim a_{\mathrm{h}}$ is essentially independent of $m_{\star}$, or equivalently of the number of particles, $N$, used to represent the galaxy. At later times, $t \gtrsim 200$ or at $a \lesssim a_{\mathrm{h}}$, the binary hardening rate becomes significantly longer and shows a clear $N$-dependence, in the sense of slower hardening for larger $N$. In this phase, the hardening of the binary requires a repopulation of the depleted orbits through collisional loss-cone repopulation, which is an $N$-dependent process (e.g., Yu 2002).

The right panels of Figure 5 show the evolution of the massive binary in a set of integrations with the same value of $N$ but for different values of $q$. From these plots we see that the dynamical friction timescale of a massive binary above $a_{\text {crit }}$ scales approximately linearly with the mass of the secondary body (see also Figure 4). This is also expected given that the dynamical friction acceleration decreases proportionally with $m$, if we neglect the logarithmic dependence of the frictional drag on $m$ through $\ln \Lambda$. As before, the massive binary orbit is observed to stall at $\approx a_{\mathrm{h}}$.

Figures 5 and 6 show that the evolution of the binary eccentricity can be divided in two distinct phases. At separations $a>a_{\text {crit }}$, the eccentricity of the binary changes steadily with time: it increases for $\gamma=(0.6,1)$ and decreases for $\gamma=2$. In the second phase, at $a<a_{\text {crit }}$, the eccentricity established at early times tends to persist, remaining approximately constant as the orbit keeps shrinking. Because $a_{\text {crit }}$ increases with $m$ (see Equation (51)), lighter inspiraling bodies have higher eccentricities by the time their orbit has shrunk to $a \approx a_{\mathrm{h}}$. This fact appears evident in the top-right panel of Figure 5 where the general trend is clearly toward higher final eccentricities for smaller $q$.

\subsection{Comparison to Analytical Predictions}

As can be seen from Figures 4-6, the evolution of $e$ and $a$ at $a>a_{\text {crit }}$ in the $N$-body simulations show good agreement with the theoretical predictions. For the cases we considered, the results of the $N$-body simulations confirm the expected qualitative result that for $\gamma \leqslant 1$, the eccentricity will increase during the inspiral while for $\gamma \geqslant 2$, the eccentricity will decrease. We conclude that the results of the $N$-body simulations support the correctness of the analytical treatment developed in Section 2 and consequently of Chandrasekhar's physical picture of dynamical friction.

Although the agreement with the theoretical prediction appears fairly good, a difference is also apparent: both $a$ and $e$ evolve more slowly in the $\mathrm{N}$-body simulations than predicted. This results in a small displacement towards the right of the corresponding analytical curves shown in the figures. The discrepancy is caused by the fact that in our analytical treatment the contribution of the stars to the gravitational potential is ignored. Consequently, the massive body moves slower relative to the $N$-body, which leads to an artificially stronger frictional drag due to the $\propto v^{-2}$ dependence that appears in Equation (11). This also explains why for smaller initial separations the agreement appears to improve-for smaller $a_{0}$ the contribution of the stars to the gravitational potential can be more safely neglected given that the potential is more strongly dominated by the primary SMBH.

We have shown that after the binary semimajor axis has decreased to $a_{\text {crit }}$, the eccentricity remains approximately constant in time. Therefore, the value of $e$ at $a_{\text {crit }}$ is also approximately the eccentricity the binary will have at the time it has decayed to $a_{\mathrm{h}}$. Below $a_{\mathrm{h}}$, the evolution becomes more uncertain. Scattering experiments typically suggest a slow but steady growth of eccentricity (Mikkola \& Valtonen 1992).

The predicted eccentricity of the massive binary at $a_{\text {crit }}$ is given in Figure 7 as a function of the binary mass ratio, different initial values of the orbital eccentricity, and for $\gamma=(0.6,1)$. In this plot we compare the theoretically predicted results with the results from the $\mathrm{N}$-body simulations, which are shown as solid triangles and which were obtained as the average value of $e$ at radii $a<a_{\text {crit }}$. The agreement with the 

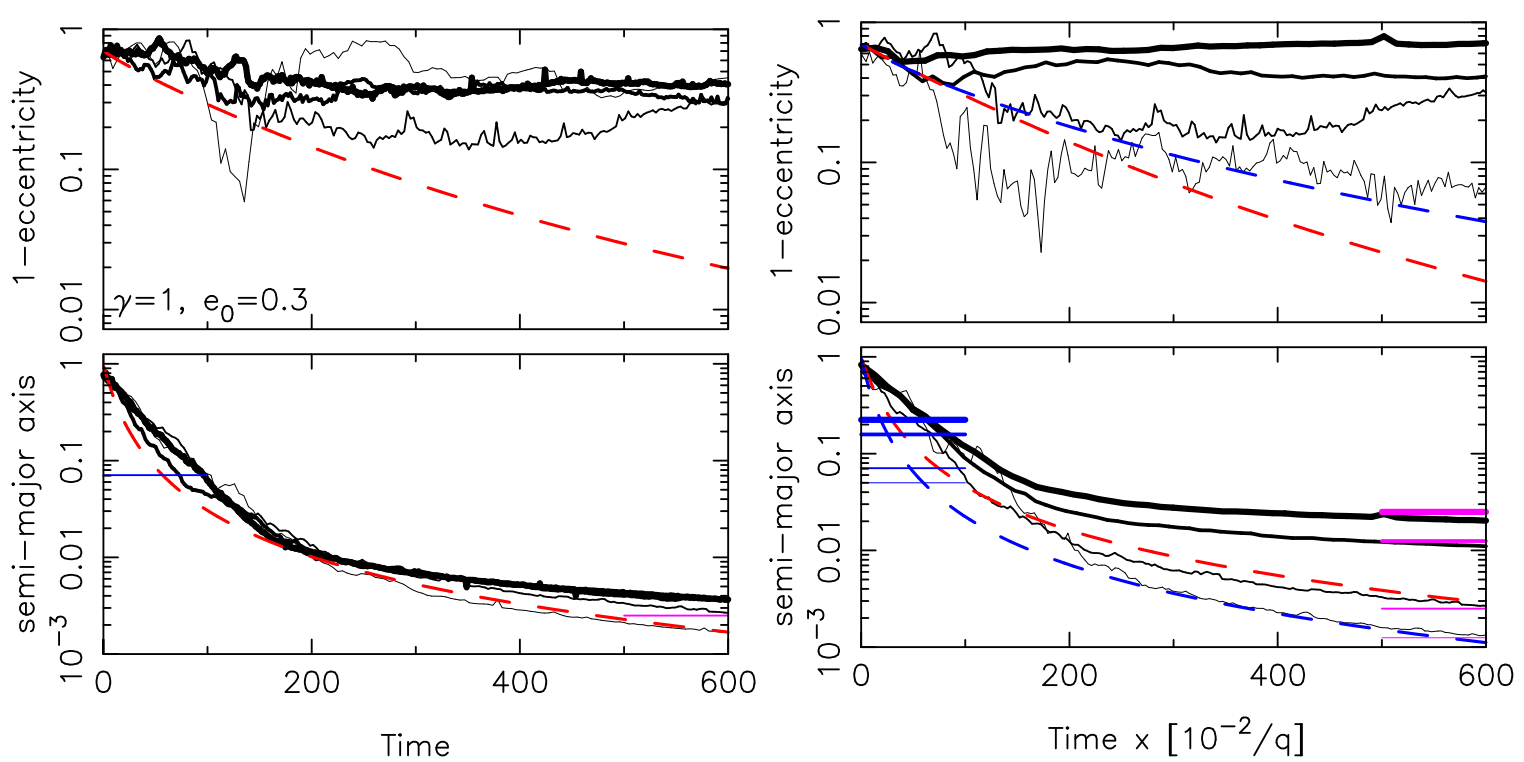

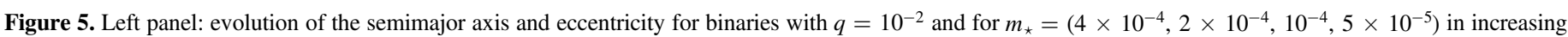

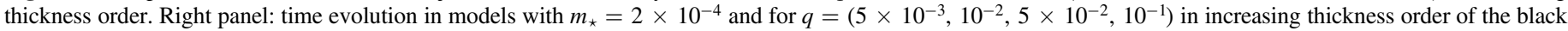

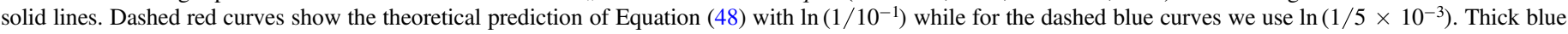

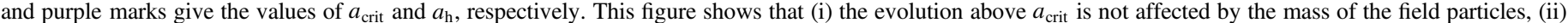
above $a_{\text {crit }}$ dynamical friction leads towards higher eccentricities, and (iii) below $a_{\text {crit }}$ the eccentricity remains approximately constant with time.

analytical predictions is again good. We see that for low massratio binaries and a moderate initial eccentricity, $e_{0} \gtrsim 0.3$, the binary will reach $e \gtrsim 0.9$ by the time it has decayed to $a_{\text {crit }}$. The results of this analysis indicate that due to dynamical friction, the eccentricity of a comparatively low-mass test body moving in the center of a massive galaxy will be high during the time it spends inside the sphere of influence of the central SMBH.

The eccentricity evolution itself is especially important in the case of SMBH binaries since the energy losses due to gravitational-wave emissions depend strongly on $e$. How much the binary must shrink by stellar-dynamical processes before gravitational-wave emission takes over is very sensitive to the eccentricity of the binary. In what follows we apply the formalism developed in Section 2 and confirmed with $N$-body simulations to describe the evolution of $\mathrm{SMBH}$ binaries in early-type galaxies.

\section{Formation of SMBH Binaries}

In what follows, we discuss the implications of our results in relation to the dynamics of the SMBH binaries that are believed to form during the merger of galaxies.

The evolution of an SMBH binary can be divided into three phases: (i) the large-scale orbital decay of the satellite galaxy from a distance of order the primary galaxy half-mass radius, or the effective radius; this phase ends when the separation between the two SMBHs reaches the primary SMBH influence radius, $\sim r_{\text {infl }}$; (ii) the inspiral of the satellite galaxy $\mathrm{SMBH}$ within the sphere of influence of the primary SMBH. In this phase, the motion of the secondary SMBH is approximately Keplerian and the evolution of its orbit can be described by Equations (28)-(33); (iii) when the binary's binding energy reaches $\sim M_{.} \sigma^{2}$, the two SMBHs form a "hard binary" at the center of the merger product. At this stage, stars that intersect the SMBH binary are ejected from the system. We anticipate here that the evolution at this stage is likely to be efficient, leading to the hardening of the binary and to its final coalescence on a timescale $\lesssim 1 \mathrm{Gyr}$.

In this section we give expressions to calculate the characteristic timescales associated with each of the three SMBH binary evolutionary stages and discuss the connection between dynamical friction and the formation of stalled SMBH satellites in luminous galaxies.

\subsection{Large-scale Orbital Decay}

Modeling the main galaxy as a singular isothermal sphere, the timescale for a satellite SMBH of mass $m$ to decay towards the center is (Binney \& Tremaine 1987)

$$
T_{\star}^{\mathrm{bare}}=17 \frac{6.6}{\ln \Lambda}\left(\frac{R_{\mathrm{e}}}{10 \mathrm{kpc}}\right)^{2}\left(\frac{\sigma}{300 \mathrm{~km} \mathrm{~s}^{-1}}\right)\left(\frac{10^{8} M_{\odot}}{m}\right) \mathrm{Gyr},
$$

where $R_{\mathrm{e}}$ is the effective radius of the main galaxy.

Some previous work made use of Equation (53) to describe the formation and evolution of SMBH binaries during galaxy mergers (e.g., McWilliams et al. 2014). However, Equation (53) can lead to a significant overestimate of the real dynamical friction timescale and to erroneous conclusions about the survival timescale of $\mathrm{SMBH}$ pairs at the scale of $R_{\mathrm{e}}$. Equation (53) neglects the fact that the satellite $\mathrm{SMBH}$ is brought in during the course of a galaxy merger and it will retain some of its host galaxy's stars until late in the merger process. Considering the stellar mass bound to the inspiraling SMBH leads to a significantly shorter timescale for the formation of a bound pair.

By assuming a strict proportionality between the mass of the stellar bulge of the satellite galaxy, $M_{\mathrm{s}}$, and the mass of its central SMBH $M_{\mathrm{s}}=10^{3} m$ (Merritt \& Ferrarese 2001), and 

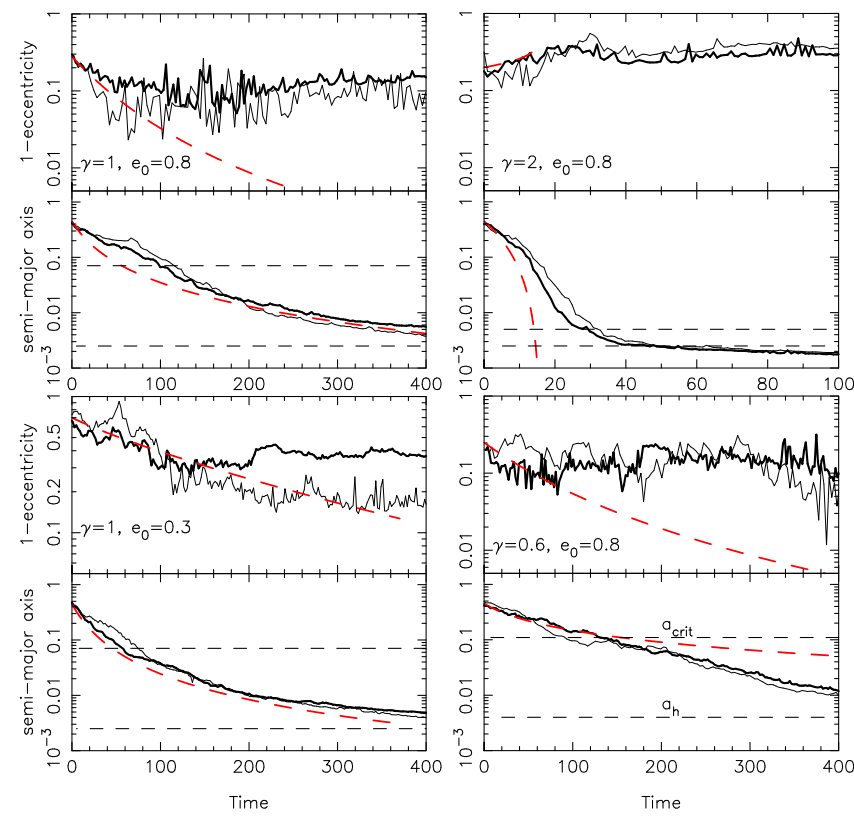

Figure 6. Evolution of massive binaries with $q=10^{-2}$ in models with $m_{\star}=10^{-4}$ (thin lines) and $2 \times 10^{-4}$ (thick lines). Dashed red lines show the theoretical predictions based on Equation (48). Dashed black lines depict the values of $a_{\text {crit }}$ and $a_{\mathrm{h}}$.

replacing $m$ with $M_{\mathrm{s}}$ in Equation (53) gives

$$
T_{\star, 1}^{\mathrm{gx}}=0.06 \frac{2}{\ln \Lambda^{\prime}}\left(\frac{R_{\mathrm{e}}}{10 \mathrm{kpc}}\right)^{2}\left(\frac{\sigma}{300 \mathrm{~km} \mathrm{~s}^{-1}}\right)\left(\frac{10^{8} M_{\odot}}{m}\right) \mathrm{Gyr},
$$

where the argument of the Coulomb logarithm is taken to be $\Lambda^{\prime}=2^{3 / 2} \sigma / \sigma_{\mathrm{s}}$, with $\sigma_{\mathrm{s}}$ the stellar velocity dispersion of the secondary galaxy.

Equation (54) does not consider that the satellite galaxy can be stripped of its stars due to the strong tidal field of the primary galaxy. Following Binney \& Tremaine (1987), we assume that the satellite galaxy can also be modeled as a singular isothermal sphere, so that its mass is related to its velocity dispersion through the relation

$$
M_{\mathrm{s}}(r) \approx \frac{1}{2 G} \alpha^{2} \sigma_{\mathrm{s}}^{2} r_{t} .
$$

Setting the Hill radius $r_{t}=\left(G M_{\mathrm{m}} r^{2} / 4 \sigma^{2}\right)^{1 / 3}$ with $M_{\mathrm{m}}$ the mass of the main galaxy and $\alpha=2$ as appropriate for a sharp truncation, the dynamical friction timescale becomes

$$
\begin{aligned}
T_{\star, 2}^{\mathrm{gx}}= & 0.15 \frac{2}{\ln \Lambda^{\prime}}\left(\frac{R_{\mathrm{e}}}{10 \mathrm{kpc}}\right)\left(\frac{\sigma}{300 \mathrm{~km} \mathrm{~s}^{-1}}\right)^{2} \\
& \times\left(\frac{100 \mathrm{~km} \mathrm{~s}^{-1}}{\sigma_{\mathrm{s}}}\right)^{3} \mathrm{Gyr} .
\end{aligned}
$$

A good approximation of the timescale for a secondary $\mathrm{SMBH}$ to decay from $R_{\mathrm{e}}$ to the influence radius of the primary $\mathrm{SMBH}$ is

$$
T_{\star}=\max \left(T_{\star, 1}^{\mathrm{gx}}, T_{\star, 2}^{\mathrm{gx}}\right) .
$$

\subsection{Dynamical Friction of a Bound Pair}

As the secondary SMBH enters the sphere of influence of the primary, the SMBHs are bound to each other, and the formulae given above, which are only strictly valid for a Maxwellian distribution of velocities and a self-gravitating cluster, can no longer apply. Nevertheless, most works in the past (e.g., Kelley et al. 2016) have neglected such a complication and applied Equation (53) until $a_{\mathrm{h}}$.

Although such a simplification is reasonable for major mergers, we find that for $q \lesssim 0.1$ it necessarily leads to an erroneous evaluation of the binary evolution timescale as well as the evolution of its orbital eccentricity.

Using Equation (28), it can be shown that the characteristic dynamical friction timescale to decay from the primary $\mathrm{SMBH}$ influence radius, $r_{\text {infl }}$, to a much shorter separation, $r=\chi r_{\text {infl }}$, is

$$
\begin{aligned}
T_{\bullet}^{\text {bare }}= & 1.5 \times 10^{7} \frac{[\ln \Lambda \alpha+\beta+\delta]^{-1}}{(3 / 2-\gamma)(3-\gamma)}\left(\chi^{\gamma-3 / 2}-1\right) \\
& \times\left(\frac{M \cdot}{3 \times 10^{9} M_{\odot}}\right)^{1 / 2}\left(\frac{m}{10^{8} M_{\odot}}\right)^{-1}\left(\frac{r_{\text {infl }}}{300 \mathrm{pc}}\right)^{3 / 2} \text { years. }
\end{aligned}
$$

The coefficients $\alpha, \beta$, and $\delta$ can be easily computed from Equations (17)-(19), assuming a circular orbit, i.e., setting $\xi=1$ in these equations as justified by the fact that the orbital decay timescale is not significantly affected by the binary orbital eccentricity.

Equation (58) does not consider that part of the host galaxy can remain bound to the secondary SMBH even inside $r_{\text {infl }}$. Assuming as before that the satellite galaxy can be modeled as a singular isothermal sphere, then the timescale to decay from $r_{\text {infl }}$ to a smaller radius $r=\chi r_{\text {infl }}$ can be obtained by replacing $m$ with $M_{\mathrm{s}}(r)$, where now $r_{t} \approx\left(M_{\mathrm{s}} / 2 M_{0}\right)^{1 / 3} r$. In this case, Equation (28) leads to

$$
\begin{aligned}
T_{\bullet}^{\mathrm{gx}}= & 1.2 \times 10^{7} \frac{\left[\ln \Lambda^{\prime} \alpha+\beta+\delta\right]^{-1}}{(3-\gamma)^{2}}\left(\chi^{\gamma-3}-1\right) \\
& \times\left(\frac{M \cdot}{3 \times 10^{9} M_{\odot}}\right)\left(\frac{100 \mathrm{~km} \mathrm{~s}^{-1}}{\sigma_{\mathrm{s}}}\right)^{3} \text { years. }
\end{aligned}
$$

The decay timescale from the influence radius of the primary $\mathrm{SMBH}$ is then

$$
T_{\bullet}=\min \left(T_{\bullet}^{\mathrm{bare}}, T_{\bullet}^{\mathrm{gx}}\right) .
$$

Note that Equation (60) is strictly valid only until the secondary hole reaches $a_{\text {crit }}$ (see Equation (51)). Below this radius, the central cusp starts to be significantly modified by the secondary SMBH (Baumgardt et al. 2006) and time-dependent galaxy models are required in order to describe the details of the evolution of the binary orbit (Antonini \& Merritt 2012). Here we ignore such complications and set $\chi=a_{\text {crit }} / r_{\text {infl }}$. Our choice is clearly conservative.

In order to quantify the error one would make by employing the standard Chandrasekhar formula, we plot in Figure 8 the dynamical friction timescale from Equation (58) with $\beta=\delta=0$ divided by $T$. For $\gamma \lesssim 1$ the standard Chandrasekhar theory can lead to significant deviations from our more accurate formulation. Neglecting the fast-moving stars' contribution leads to an overestimate of the dynamical friction timescale that can be longer than $T$. by about one order of magnitude for $\gamma \approx 0.5$. In 


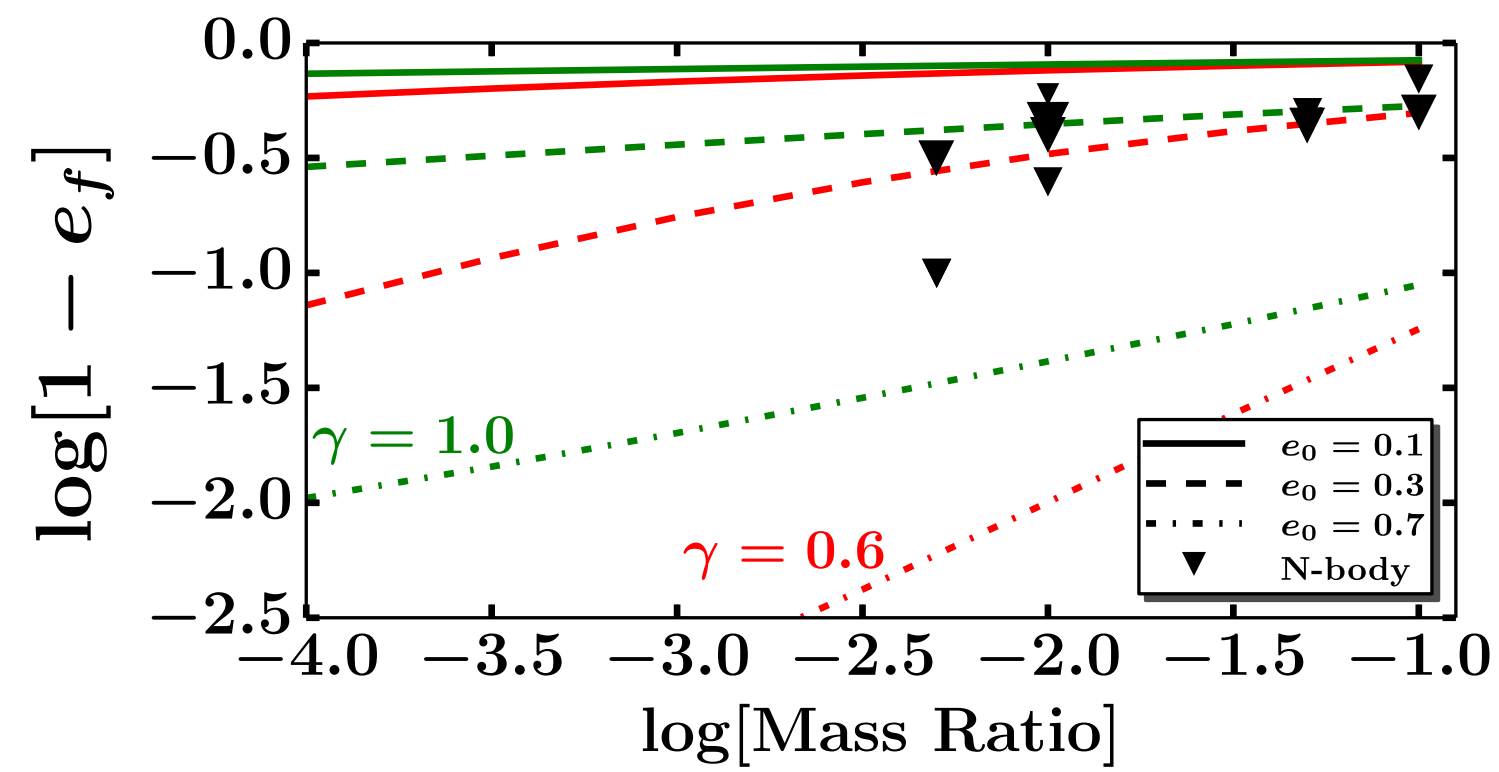

Figure 7. Eccentricity of the massive binary at the moment the inspiraling body reaches $a_{\text {crit }}$ as a function of the binary mass ratio. Black triangles represent the results from the $N$-body simulations shown in Figure 5, where $\gamma=1, e_{0}=0.3$, and $a_{0}=1$. The values of $e$ from these simulations are averaged values at $a \leqslant a_{\text {crit }}$. Increasing marker size indicates a larger number of particles, i.e., lower $m_{\star}$.

Figure 8 we also compare our estimate to that obtained by assuming that the velocity distribution of the field stars follows a Maxwellian distribution. This latter approximation underpredicts the decay timescale by about one order of magnitude for $\gamma \approx 0.5$. For $\ln \Lambda \gtrsim 6$ and/or $\gamma \gtrsim 1$ both approximations give a good estimate of the decay timescale, as the contribution of the fast stars to the drag force is smaller in this case.

\subsection{Hardening}

The phase of binary evolution determined by dynamical friction comes to an end when the binary's semimajor axis reaches $\sim a_{\mathrm{h}}$. Gravitational encounters will continue supplying stars to the binary at a rate that depends on the host galaxy morphology.

Dry mergers between luminous galaxies result in triaxial remnants, which leads to an efficient hardening of the binary (Khan et al. 2011; Vasiliev et al. 2014). After a major merger, a galaxy is likely to retain some degree of triaxiality or flattening during its subsequent evolution. The timescale to decay from $a_{\mathrm{h}}$ to coalescence is (Vasiliev et al. 2015)

$$
\begin{gathered}
T_{\mathrm{h}} \approx 1.2 \times 10^{9}\left(\frac{r_{\mathrm{infl}}}{300 \mathrm{pc}}\right)^{\frac{10+4 \psi}{5+\psi}},\left(\frac{M \cdot+m}{3 \times 10^{9} M_{\odot}}\right)^{\frac{-5-3 \psi}{5+\psi}}, \\
\phi^{\frac{-4}{5+\psi}}\left(\frac{4 q}{(1+q)^{2}}\right)^{\frac{3 \psi-1}{5+\psi}} p(e) \text { years, }
\end{gathered}
$$

with

$$
\begin{aligned}
p(e) & =\left(1-e^{2}\right)\left[k+(1-k)\left(1-e^{2}\right)^{4}\right], \\
k & =0.6+0.1 \log \left([M \bullet+m] / 3 \times 10^{9} M \odot\right) .
\end{aligned}
$$

The parameters $\phi$ and $\psi$ parameterize the evolving hardening rate with values estimated from Monte Carlo simulations. In what follows we adopt $\phi=0.4$ and $\psi=0.3$, which are the values derived by Vasiliev et al. (2015) for triaxial galaxies.

We describe how much the binary must shrink by stellardynamical processes before gravitational-wave emission takes over using the ratio (Vasiliev et al. 2015):

$$
\begin{aligned}
\frac{a_{\mathrm{h}}}{a_{\mathrm{GW}}} \approx & 55 \times\left(\frac{r_{\mathrm{infl}}}{30 \mathrm{pc}}\right)^{\frac{5}{10}}\left(\frac{M \cdot+m}{10^{8} M_{\odot}}\right)^{-\frac{5}{10}} \\
& \times f(e)^{\frac{1}{5}}\left(\frac{4 q}{(1+q)^{2}}\right)^{\frac{4}{5}},
\end{aligned}
$$

with

$$
f(e)=\frac{(1-e)^{-\frac{7}{2}}}{1+\frac{73}{24} e^{2}+\frac{37}{96} e^{4}}
$$

and $a_{\mathrm{GW}}$ the separation at which gravitational-wave radiation takes over. From Equation (64) one finds that $a_{\mathrm{h}}<a_{\mathrm{GW}}$ for $q \approx 10^{-3}$ and moderate eccentricities. Binaries with mass ratio lower than this value never enter the hardening phase. The quoted approximation formula therefore breaks down in this case because these systems are never in a properly stellardynamical hardening regime. These binaries transit directly from the dynamical friction phase to the phase where gravitational-wave radiation leads to their rapid coalescence. Accordingly, in what follows we set $T_{\mathrm{h}}=0$ for $q \lesssim 10^{-3}$.

Equation (61) implies that stellar-dynamical interactions are able to drive the binary to coalescence on a timescale $\leqslant 1 \mathrm{Gyr}$ in any triaxial galaxy, and that the coalescence timescale weakly depends on the mass of the binary and its mass ratio. Coalescence times also depend quite significantly on the binary eccentricity falling in the range from a few gigayears for almost circular binaries to $\approx 10^{8}$ years for very eccentric ones.

We note that Equation (61) was derived for major mergers, $q \gtrsim 0.1$. However, additional simulations performed using the Monte Carlo code RAGA (Vasiliev 2015) showed that Equation (61) works remarkably well for all binaries with $q \gtrsim 10^{-2}$. At $q \approx 10^{-3}$, Equation (61) starts to break down, but even for $q=10^{-3}$ it overestimates by only a factor of $\approx 3$ the binary's coalescence timescale (E. Vasiliev 2017, private communication). Hence, unless otherwise specified, in what 


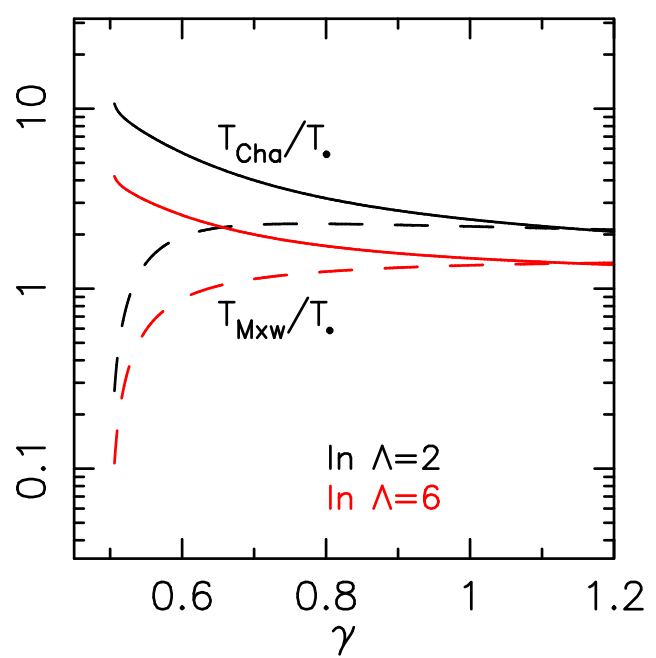

Figure 8. Solid lines give the dynamical friction timescale to reach $a_{\text {crit }}, T_{\text {Cha }}$, derived from Equation (58) by setting $\beta=\delta=0$ and divided by our more accurate estimate $T$. Dashed lines are the dynamical friction timescale, $T_{\mathrm{Mxw}}$, computed using the Maxwellian approximation and divided by $T$. This calculation quantifies the error one would make by employing the standard Chandrasekhar formula in which the contribution of the fast stars is neglected, or by assuming that the velocities of the field stars follow a Maxwellian distribution.

follows we adopt Equation (61) in the full range of mass ratios $10^{-3} \leqslant q \leqslant 1$. The fact that $T_{\mathrm{h}}$ is only an order of magnitude estimate for the lowest mass ratio binaries we considered does not affect our calculations below, given that the total lifetime of these binaries is largely dominated by their dynamical friction timescale.

Finally, we note that the assumption of triaxiality in the fieldstar distribution made in Equation (61) is obviously inconsistent with the spherical density model used to compute the dynamical friction timescales above. However, galaxy mergers produce remnants with deviations from isotropy that are small both in velocity and configuration space (Vasiliev et al. 2015). Hence, we might expect our estimates of the dynamical friction timescales to give a reasonable approximation.

\subsection{SMBH Binary Formation in Early-type Galaxies}

Here we estimate the timescales associated with the three stages of binary evolution defined above for real galaxies and consider the possibility of "stalled" mergers in these systems, where a smaller satellite SMBH resides in the outer regions of the main host galaxy. We focus on massive early-type galaxies because (i) galaxy mergers are thought to play a crucial role in the late growth of these systems and (ii) these galaxies are often observed to have extended density profile cores within the sphere of influence of the $\mathrm{SMBH}$, which implies a long dynamical friction timescale. For these reasons, luminous early-type galaxies are likely hosts of stalled satellite SMBHs. On the other hand, our results imply that in low-mass ellipticals, SMBH binaries have a short timescale, i.e., shorter inspiral times, possibly in the decreasing or near constant eccentricity regime.

We start by considering the case of a widely studied massive galaxy such as M87, for which $M_{\bullet} \approx 6.15 \times 10^{9} M_{\odot}$, $\sigma \approx 330 \mathrm{~km} \mathrm{~s}^{-1}$ (Kormendy \& Ho 2013), $R_{\mathrm{e}} \approx 9 \mathrm{kpc}$ (Lauer et al. 1995), and $r_{\text {infl }} \approx 300$ pc (Marconi \& Hunt 2003). Given these structural parameters, the calculation of the decay timescales can be simply performed by assuming that the secondary galaxy velocity dispersion is related to the mass of its central SMBH through the $M \cdot-\sigma$ relation defined below in Equation (69) setting $z=0$ (Ferrarese \& Merritt 2000; Gebhardt et al. 2000)

An additional parameter that needs to be defined is $\gamma$, the slope of the deprojected spatial density profile of stars within $r_{\text {infl }}$. We adopt the two representative values $\gamma=0.6$ and 1 , as motivated by the facts that the M87 density profile is observed to be nearly flat inside $r_{\text {infl }}$ and values $\gamma \leqslant 0.5$ are excluded by our assumption of kinematical isotropy.

The left panel of Figure 9 gives the orbital decay timescale as a function of the satellite SMBH mass inside M87 at the different stages of the evolution of the massive binary. The dynamical friction timescale to decay from the effective radius to the $\mathrm{SMBH}$ influence radius, $T_{\star}$, is shorter than $\approx 10^{9}$ years for $m \gtrsim 5 \times 10^{6}$, implying that SMBH binaries are unlikely to stall near $R_{\mathrm{e}}$. The hardening timescale to reach coalescence from the hard binary separation is quite short, $\lesssim 10^{9}$ years, even for the moderate eccentricity we adopted, $e=0.3$. The dynamical friction timescale to decay from $r_{\text {infl }}$ to $a_{\mathrm{h}}$ can instead be extremely long. For $m \lesssim 10^{8} M_{\odot}, T$. becomes longer than either $T_{\star}$ or $T_{\mathrm{h}}$, and it is longer than $10 \mathrm{Gyr}$ for $m \lesssim 10^{6} M_{\odot}$ $\left(m \lesssim 10^{7} M_{\odot}\right)$ when $\gamma=1(\gamma=0.6)$.

In the right panel of Figure 9, we show the timescale of orbital decay $T_{\star}$ for the sample of 31 core-Sérsic early-type galaxies in Dullo \& Graham (2015; purple points). These systems are bright elliptical and lenticular galaxies with extended density profile cores. Dullo \& Graham (2015) give the measured structural parameters of these galaxies, including $R_{\mathrm{e}}$ and $\sigma$, which we used to compute $T_{\star}$. This timescale is plotted as a function of the primary SMBH mass for $q=10^{-3}$. Larger symbols are systems for which a direct $\mathrm{SMBH}$ measurement is available in the literature (Kormendy \& Ho 2013). The resulting $T_{\star}$ is $\lesssim 10^{9}$ years. The hardening timescale $T_{\mathrm{h}}$ (dotted-dashed line) also appears to be quite short $\lesssim 10^{9}$ years, and, as expected, weakly dependent on the primary SMBH mass. Finally, the hatched red region gives $T_{0}$, which was computed through Equation (60) by setting (e.g., Merritt et al. 2009)

$$
r_{\mathrm{infl}} \approx 35\left(\frac{M .}{10^{8} M_{\odot}}\right)^{0.56} \mathrm{pc},
$$

with $M$. given by Equation (69) at $z=0$.

The analysis shown in the right panel of Figure 9 confirms and generalizes our statement that the dynamical friction timescale inside the influence radius of an SMBH can become of the order of the Hubble time in luminous spheroids. We considered that $T$. can be significantly larger than either $T_{\star}$ or $T_{\mathrm{h}}$, and can become longer than the Hubble time even for relatively high mass-ratio binaries not just in the core of M87 but for most galaxies in the sample.

In Figure 10, we investigate further the dependence of the lifetime of an SMBH binary on its total mass and mass ratio. The total lifetime of an SMBH binary is given by $t_{\mathrm{L}}=T_{\star}+T_{\bullet}+T_{\mathrm{h}}$. We plot the lifetime of a massive black hole binary in the three different stages of the binary evolution as a function of the binary total mass and mass ratio. For the effective radius of the host galaxy, we use $R_{\mathrm{e}}=1.35\left(M_{\bullet} / 10^{8} M_{\odot}\right)^{0.73} \mathrm{kpc}$, where the mass dependence and normalization were obtained from the observed massradius relation of galaxies at redshift zero (Figure 7 in Forbes et al. 2008 for their sample of elliptical galaxies), and after 

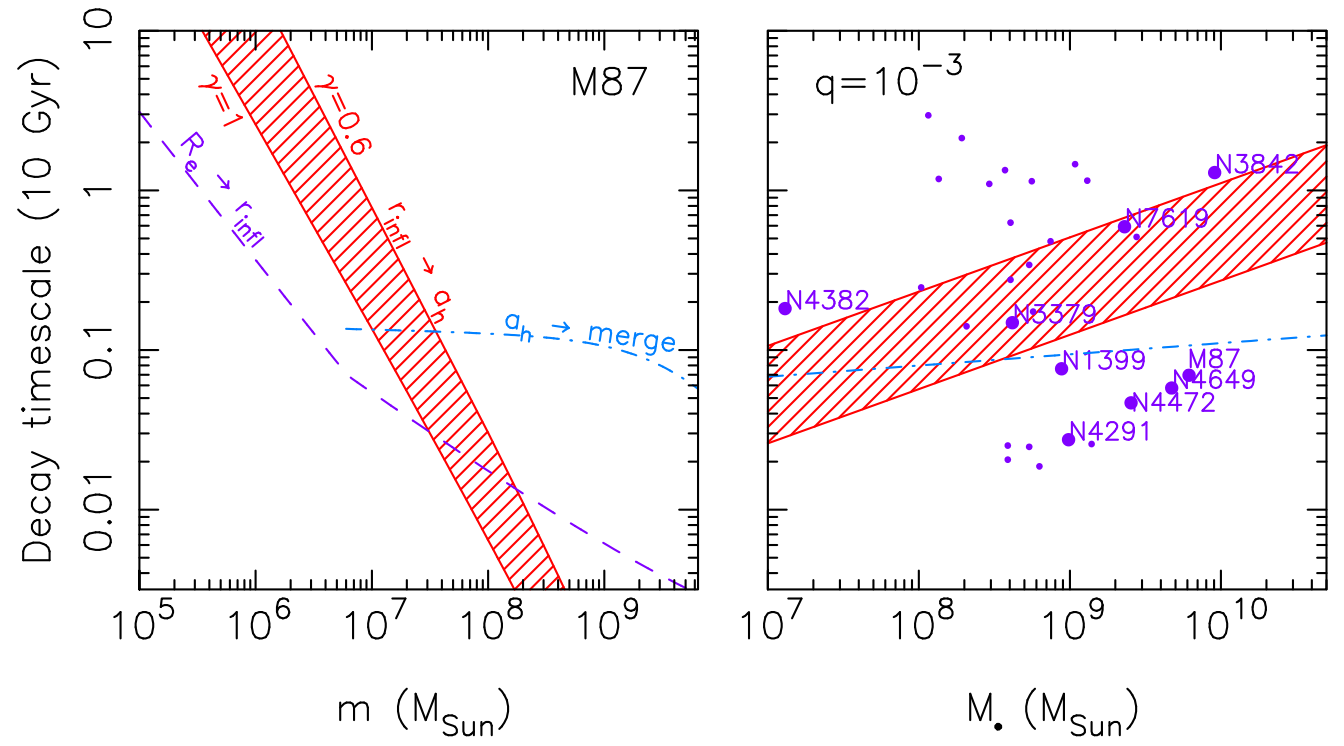

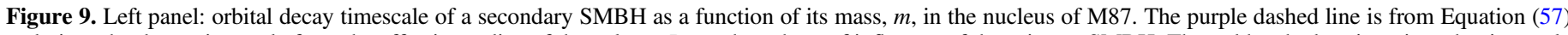

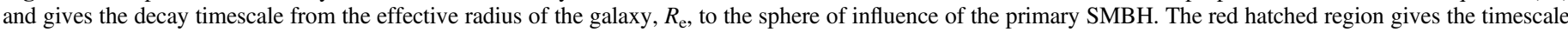

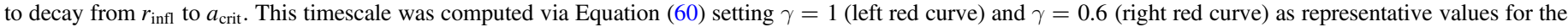

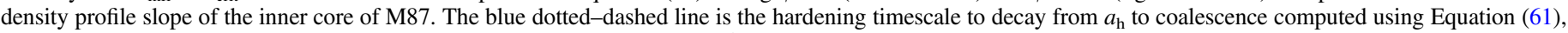

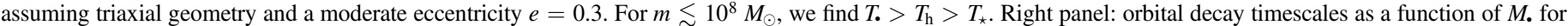

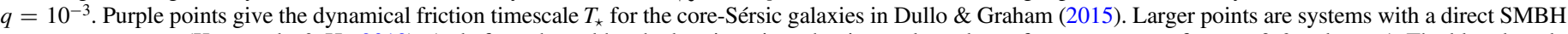

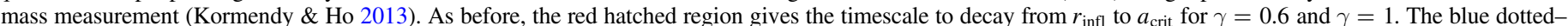
dashed line is $T_{\mathrm{h}}$.

expressing the galaxy mass as $M_{\mathrm{m}}=10^{3} M_{\bullet}$, assuming the latter relation holds at all redshifts.

Based on Figure 10, the amount of time the secondary $\mathrm{SMBH}$ spends to decay from the effective radius of the galaxy $R_{\mathrm{e}}$ to the influence radius of the central black hole is short, typically less than $\sim 3$ Gyr. Adding the time the binary spends in the dynamical friction phase until $a_{\text {crit }}$ leads to significantly longer binary lifetimes. Figure 10 shows that including the dynamical friction timescale for high total-mass and low massratio binaries expands the parameter space for long-lived binaries with lifetimes greater than $\sim 3 \mathrm{Gyr}$, while there is a considerable amount of these binaries that have lifetimes greater than $\sim 10$ Gyr. This implies that binaries with high total mass and low mass ratio are expected to live long in the evolutionary stage between the SMBH influence radius and hardening radius of the binary. Based on Figure 7, we also expect that these binaries will have high eccentricities. The final phase in the evolution of an SMBH binary before coalescence is characterized by the hardening timescale that we add in the binary total lifetime in the right panel of Figure 10. The hardening timescale contributes mostly to the lifetime of high mass-ratio binaries, always being shorter than $\approx 1 \mathrm{Gyr}$.

\section{Stalled Satellites in Minor Mergers}

In Section 4, we studied the lifetime of an SMBH binary. We found that $\mathrm{SMBH}$ binaries with high total mass and low mass ratio are long-lived. These binaries are the product of minor galaxy mergers where a smaller galaxy is accreted by a giant galaxy. If the lifetime of a massive binary exceeds the time passed from its formation redshift to the present time, the binary orbit stalls and the secondary SMBH becomes a stalled satellite. The number of stalled satellites over the Hubble time depends on the formation redshift of the binary and the rate at which galaxies in the relevant total-mass and mass-ratio range merge with each other.

The total galaxy merger rate is defined as the rate at which a galaxy with a primary SMBH of mass $M$. experiences mergers with other galaxies at a redshift $z$. The merger rate therefore depends on how the galaxy properties evolve with redshift. In order to model the evolution of mass, effective radius, and velocity dispersion of a galaxy, we follow below the redshiftdependent fitting formulae of Nipoti et al. (2012) for typical massive early-type galaxies:

$$
\begin{gathered}
\mathcal{M}(z)=M_{\bullet}(1+z)^{-0.6} M_{\odot}, \\
\mathcal{R}_{\mathrm{e}}(z)=1.35\left(\frac{M_{\bullet}}{10^{8}}\right)^{0.73}(1+z)^{-0.71} \mathrm{kpc}, \\
\sigma(z)=180\left(\frac{M_{\bullet}}{10^{8}}\right)^{0.2}(1+z)^{0.056} \mathrm{~km} \mathrm{~s}^{-1},
\end{gathered}
$$

where now $M$. indicates the central SMBH mass at redshift $z=0$. In what follows, unless otherwise specified, we will use Equations (67)-(69) as our reference model.

The differential merger rate of galaxies can be derived based on the differential fraction of galaxies with central SMBH mass $M$. at redshift $z$ that are paired with a secondary galaxy having a mass ratio in the range $q, q+d q$ and the merger timescale for a galaxy pair with a given $M$. and $q$ at a given $z$ (e.g., Kitzbichler \& White 2008; Patton \& Atfield 2008; Bundy et al. 2009; de Ravel et al. 2009; López-Sanjuan et al. 2012; Xu et al. 2012). In this paper, we use the pair fraction derived in $\mathrm{Xu}$ et al. (2012). The differential galaxy merger rate per unit mass ratio is then given by (Xu et al. 2012; Sesana 2013; Rasskazov \& Merritt 2016)

$$
\frac{d N_{\mathrm{m}}(z, q)}{d q d t}=0.044 \frac{1}{q}\left(\frac{\mathcal{M}(z)}{10^{7.7} M_{\odot}}\right)^{0.3} \frac{(1+z)^{2.2}}{1+z / 8} \mathrm{Gyr}^{-1}
$$



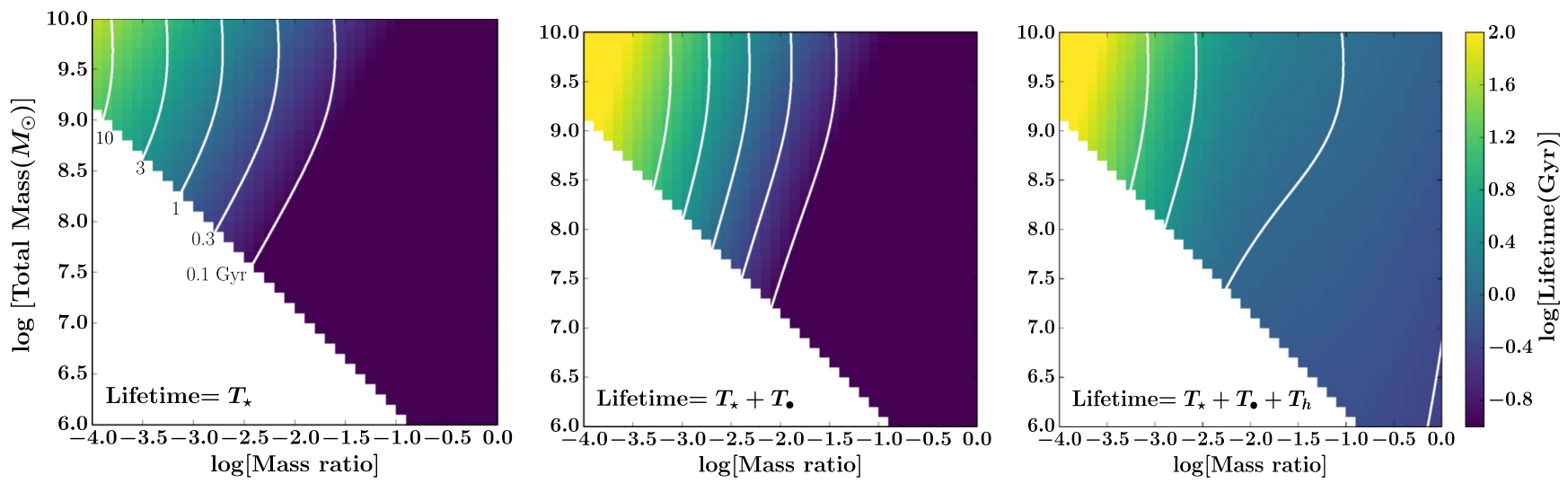

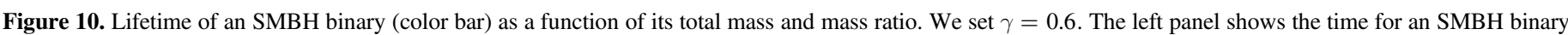

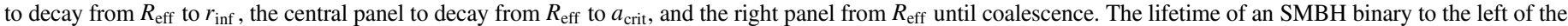

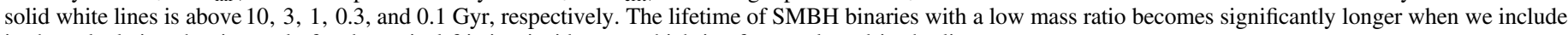
in the calculation the timescale for dynamical friction inside $r_{\mathrm{inf}}$, which is often neglected in the literature.

where $N_{\mathrm{m}}$ is the the number of mergers. In the work of Xu et al. (2012), Equation (70) refers to mergers in the data sample with maximum merger mass ratio $M . / m=2.5$. For the observed pair distribution as a function of the mass ratio $q$, a good proxy is $\propto 1 / q$ (e.g., Sesana 2013). Cosmological simulations in Rodriguez-Gomez et al. (2015) suggest that $\propto 1 / q$ is also a good proxy for all mass ratios in the range $10^{-4}<q<1$. More specifically, in Rodriguez-Gomez et al. (2015), $d N_{\mathrm{m}} / d q_{\mathrm{h}} \propto$ $q_{\mathrm{h}}^{-1.7}$, where $q_{\mathrm{h}}$ refers to the dark-matter halo mass ratio. Following the work of Baes et al. (2003) and assuming an SMBH-halo mass relation $M_{\bullet} \propto M_{\mathrm{h}}^{1.3}$ leads to $d N_{\mathrm{m}} / d q \propto q^{-1.5}$ (i.e., between the linear scaling adopted here and a quadratic scaling).

We can calculate the expected average number of stalled satellite $\mathrm{SMBHs}, N_{\mathrm{s}}$, for a galaxy with a central $\mathrm{SMBH}$ with mass $M$. by integrating the differential merger rate (70) over the relevant range of redshift $0<z<z_{\max }$ and mass ratio $q_{\text {min }}<q<q_{\text {crit }}$ :

$$
N_{\mathrm{s}}\left(M_{\bullet}\right)=\int_{0}^{z_{\max }} \int_{q_{\min }}^{q_{\mathrm{crit}}} \frac{d N_{\mathrm{m}}(z, q)}{d q d t} \frac{d t}{d z} d z d q,
$$

where $q_{\text {crit }}$ is the critical value of the mass ratio below which the lifetime of the massive binary exceeds the time that has passed from its formation until today. This was computed as the solution to the equation

$$
t_{\mathrm{L}}\left(q_{\text {crit }}, z, \gamma\right)=10 \mathrm{Gyr} \text {. }
$$

In this calculation, we set $z_{\max }=1$ because at redshifts lower than this, ellipticals contain little gas and the SMBH of massive ellipticals grows primarily through minor mergers (e.g., Haehnelt \& Kauffmann 2002). We consider secondary SMBHs with mass $\geqslant 10^{6} M_{\odot}$, i.e., $q_{\min }=10^{6} M_{\odot} / M_{\text {. }}$, central $\mathrm{SMBH}$ masses within the range $7.5<\log \left(M_{\bullet}\right)<10.0$, we use

$$
\frac{d t}{d z}=\frac{1}{H_{0}(1+z) \sqrt{\Omega_{\mathrm{M}}(1+z)^{3}+\Omega_{\Lambda}}}
$$

and assume a flat cosmology with the WMAP seven-year values for the cosmological parameters $H_{0}=70.3\left(\mathrm{~km} \mathrm{~s}^{-1}\right)$ $\mathrm{Mpc}^{-1}, \Omega_{\Lambda}=0.729$ and $\Omega_{\mathrm{M}}=0.1338$ (Komatsu et al. 2011). To give a sense of the merger inspiral efficiency we plot in Figure 11 the average number of infalling satellites for $0<z<1$ and as a function of the host galaxy SMBH mass, setting $q_{\text {crit }}=1$ in Equation (71). Note that the difference $\left(N_{\mathrm{m}}-N_{\mathrm{s}}\right)$ is the number of $\mathrm{BH}$ binaries that have reached coalescence.

In Figure 12, we plot $N_{\mathrm{s}}$ as a function of the host galaxy SMBH mass $M$. for $\gamma=0.6$ and $\gamma=1.0$. The galaxy properties are related to the mass of the $\mathrm{SMBH}$ through Equations (67)-(69), while we explore the effect of a steeper redshift dependence on the galaxy properties using a different model from Nipoti et al. (2012). We find that the expected number of stalled satellites increases with the galaxy mass, although adopting a different model for the redshift evolution of the galaxy properties does not significantly affect the number of stalled holes.

We perform the same calculation using the observed galaxy properties for the group of elliptical galaxies in Dullo \& Graham (2015) and used the redshift dependence from Equations (67)-(68). For the galaxies for which we do not have a measurement of their SMBH mass, we infer this using the $M_{0}-\sigma$ relation (69) with $z=0$. We also plot the expected number of stalled satellites based on the results of the cosmological simulation ILLUSTRIS taken from Kelley et al. (2016). More specifically, for different values of redshift in the range $0<z<1$ and for the two density profiles in Figure 12, we computed the value of $q_{\text {crit }}$. Using Figure 12 in Kelley et al. (2016), we calculated the number of stalled satellites we expect for a given total-mass binary, counting the number of mergers in the range $q<q_{\text {crit }}$. Figure 2 in Kelley et al. (2016) gives the total number of mergers in the simulation for a given total binary mass. Adding the number of stalled satellites in the various redshift ranges and dividing by the total number of mergers for a given total binary mass leads to an estimate of the average number of stalled satellites expected from the results in ILLUSTRIS. Based on Figure 12 the three different treatments seem to agree pretty well with each other. For massive galaxies, we expect a few stalled satellites within their inner cores.

The question on what spatial scale the satellites stall still remains unanswered. For this purpose, we computed the number of black holes that stall when setting $t_{\mathrm{L}}=T_{\star}$. With this choice, for the galaxies in our sample with $10^{8} M_{\odot} \lesssim$ $M . \lesssim 10^{9} M_{\odot}$, i.e., N6876, N4073, and N4696, the number of stalled satellites was roughly unchanged, demonstrating that in these specific galaxies the satellites are expected to reside at 
$\sim R_{\mathrm{e}}$. For $\mathrm{SMBH}$ masses larger than this, however, setting $t_{\mathrm{L}}=T_{\star}$ gave a much smaller number of stalled binaries in most galaxies we considered. We conclude that for the assumed model of hard binary inspiral in a triaxial potential, nearly all of the SMBHs in the most massive systems we considered stall at radii $r_{\mathrm{h}} \lesssim r \lesssim r_{\text {infl }}$.

To address more precisely where the stalling might occur, we plot in Figure 13 the time evolution of the semimajor axis of a secondary SMBH for two mass ratios $q=\left(10^{-3}, 10^{-4}\right)$ and for $\gamma=0.6$. The figure shows that the orbital decay significantly slows down at $\approx 0.1 r_{\text {infl }}$, which for a typical giant elliptical galaxy corresponds to galactocentric distances of tens of parsecs.

\subsection{Observational Evidence}

The observational evidence of small-orbit SMBH binaries is still scarce. Electromagnetic tracers of post-merger galaxy cores are hard to identify. This makes the study of the postmerger dynamics of binary SMBH systems difficult; pairs of SMBHs are usually observed during the early stages in their dynamical evolution when still at $\sim \mathrm{kpc}$ separations. Although observing SMBH binaries at parsec scales is challenging since they cannot be spatially resolved in optical and X-ray, more work in detecting SMBH binaries at subkiloparsec scales $(\lesssim 100 \mathrm{pc})$ is needed since discovering such systems and obtaining a census of their population properties would serve as a test of galaxy evolution models and would provide valuable constraints for stellar and gas dynamical models for the decay of the binary orbit.

If accretion is triggered along the course of the merger, direct evidence of SMBH binaries comes from the presence of active galactic nuclei (AGNs). Accretion by SMBHs can give rise to a rich phenomenology that goes from dual to binary and off-set AGNs, in the radio-loud or quite mode, according to their dynamics, habitat, and merger type. Specifically, if only one $\mathrm{SMBH}$ is accreting, it will be visible as an off-center AGN accreting from a small disk. If both binary components of the SMBH binary accrete, then a dual or double AGN might be observed.

In this work, we are interested in mergers that occur in gaspoor environments where collision processes are at play. Core galaxies are promising systems in which to search for $\mathrm{SMBH}$ binaries since they have experienced a number of minor mergers during their lifetime and are minimally affected by extinction. For example, a number of displaced SMBHs have been observed as off-set AGNs in host elliptical galaxies (e.g., Lena et al. 2014). A characteristic example is the galaxy M87 (NGC 4486) which has a measured $7.7 \pm 0.3 \mathrm{pc}$ projected displacement of the photocenter relative to the AGN (Batcheldor et al. 2010; Janowiecki et al. 2010). As a fraction of the rather large core radius $r_{\mathrm{c}}$, the weighted mean displacement is only $\approx 0.01 r_{\mathrm{c}}$. The expected observed displacement $\Delta r$ of the primary $\mathrm{SMBH}$ is defined as $\Delta r=q R /(1+q)$ for $q=10^{-4}$ and $q=10^{-3}$, with $R$ the orbital separation between the two SMBHs. Based on Figure 13, the secondary SMBHs are expected to stall at a distance $a_{\text {stall }} \approx 0.1 r_{\text {infl }}$. At this radius we have $\Delta r \approx q a_{\text {stall }} /(1+q)$, which gives $\Delta r / r_{\text {infl }} \sim 10^{-4}$ $\left(q=10^{-3}\right)$ and $\Delta r / r_{\text {infl }} \sim 10^{-5}\left(q=10^{-4}\right)$. These values are small and not consistent with the observed off-center displacements mentioned in Lena et al. (2014) which are of order $\sim 10^{-1}-10^{-2}$ for the massive elliptical galaxies shown in Figure 13 following the sample of Lena et al. (2014). However,

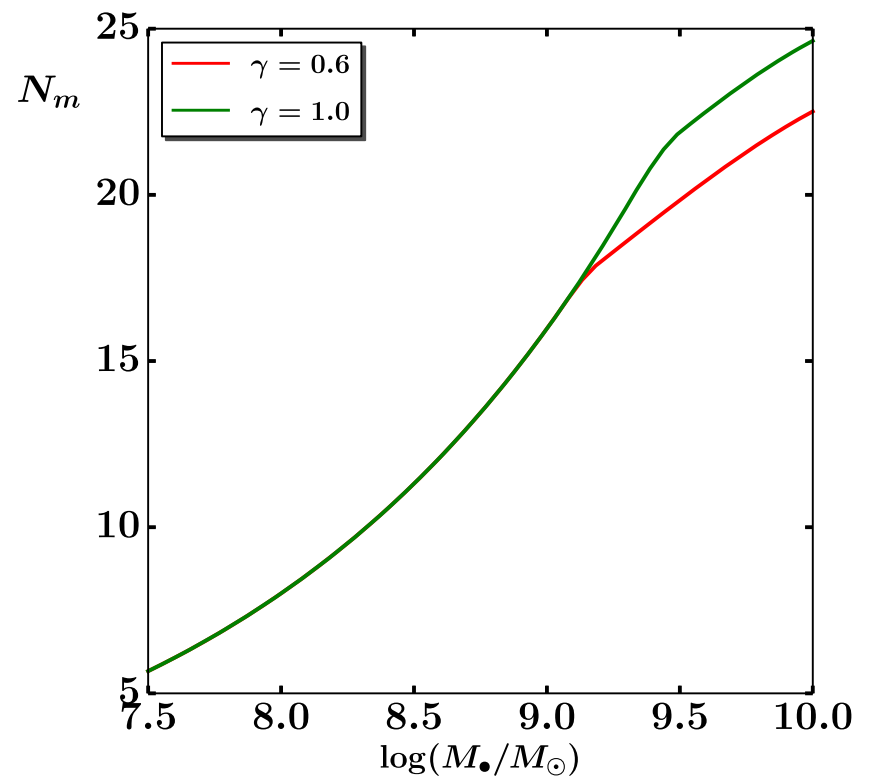

Figure 11. Average number of infalling satellites for $0<z<1$ and as a function of the host galaxy SMBH mass for two different stellar cusp density profiles.

if we assume that the secondary, rather than the primary, SMBH is accreting, then the predicted offsets, in this case the galactocentric distance of the satellite $\mathrm{SMBH}$, appear to be consistent at least with those observed in NGC 4278 and NGC 5846.

Although difficult to spatially resolve in the optical and $\mathrm{X}$-ray, giant ellipticals often host strong radio sources. For example, a pair of AGNs within the elliptical host galaxy $0402+379$ and with a projected separation of $7.2 \mathrm{pc}$ has recently been discovered (Rodriguez et al. 2006, 2009; BurkeSpolaor 2011). This is the closest binary SMBH yet discovered within a core elliptical and may be the tip of the iceberg of SMBH binaries with parsec-scale separations. Binary AGNs within host core ellipticals at subkiloparsec separations like the one in Rodriguez et al. (2006) could be explained as stalled holes in a slowly evolving orbit inside low-density cores.

Early observations have suggested the presence of double nuclei in core elliptical galaxies with the second nucleus at offcenter subkiloparsec separations. A characteristic example is the double nucleus of the core elliptical galaxy NGC 5419 with the second nucleus at an off-center separation $\sim 70 \mathrm{pc}$ (Mazzalay et al. 2016). The projected separation between the two nuclei is smaller than the estimated SMBH influence radius $\left(r_{\text {infl }} \approx 100 \mathrm{pc}\right)$ and much smaller than the core radius $\left(r_{\mathrm{c}} \approx 500 \mathrm{pc}\right)$. The same appears to be true in the case of NGC 4696, which has a secondary nucleus at $\sim 30$ pc from the center (Laine et al. 2003), which is slightly smaller than the estimated SMBH influence radius $\left(r_{\text {infl }} \approx 40 \mathrm{pc}\right)$ and much smaller than the core radius $\left(r_{\mathrm{c}} \approx 250 \mathrm{pc}\right)$. These features in NGC 4696 have been interpreted as evidence of a recent minor merger with a gas-rich galaxy (Sparks et al. 1989; Farage et al. 2010). The case of NGC 6876, which has a double nucleus at $\sim 30$ pc from the center (Lauer et al. 2002; Dullo \& Graham 2012), is similar. This is slightly smaller than the estimated SMBH influence radius $\left(r_{\mathrm{infl}} \approx 40 \mathrm{pc}\right)$ and much smaller than the galaxy inner core radius $\left(r_{\mathrm{c}} \approx 119 \mathrm{pc}\right)$.

More recent observations suggest the presence of multiple nuclei located (in projection) within the core elliptical galaxy 

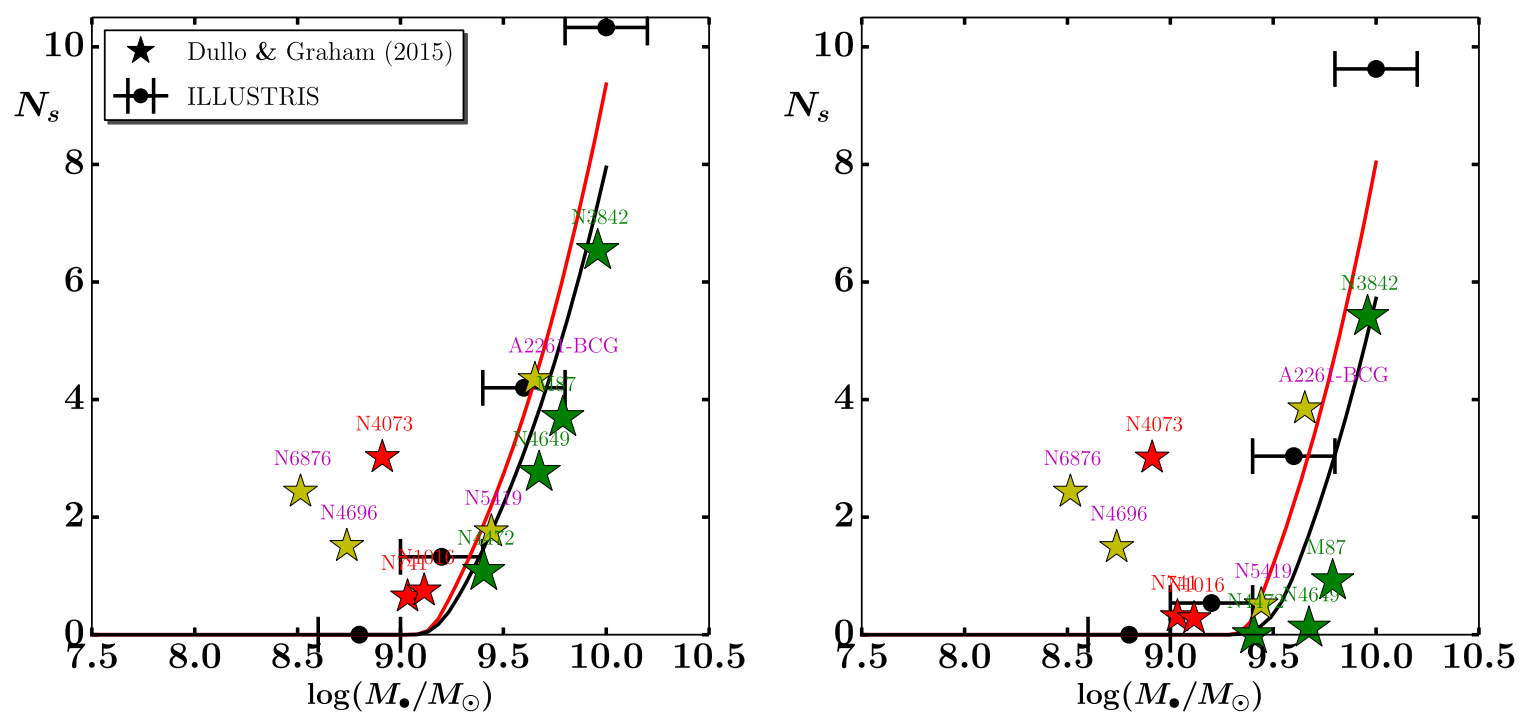

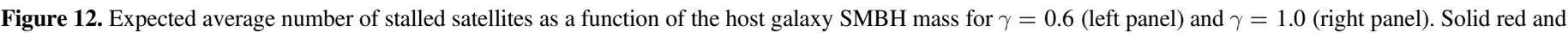

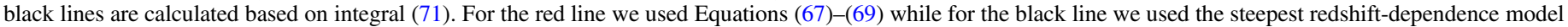

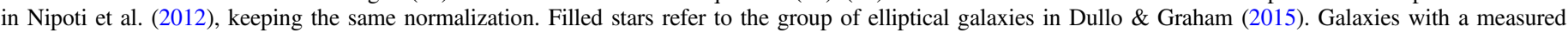

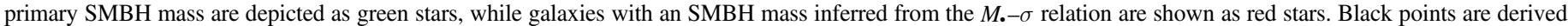

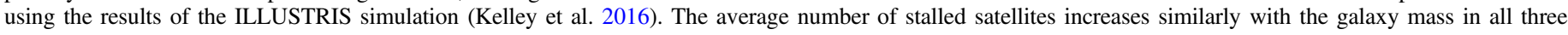

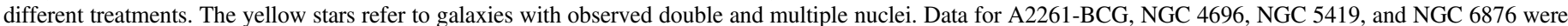
taken from Postman et al. (2012), Arnalte Mur et al. (2006), Mazzalay et al. (2016) and Lauer et al. (2002), and Dullo \& Graham (2012), respectively.

A2261-BCG (Bonfini \& Graham 2016). The estimated mass of the core and the central SMBH in A2261-BCG is $M_{\text {core }} \sim M_{0} \sim 2 \times 10^{10}$. This implies that if an SMBH is present at the center of this galaxy, then such nuclei are residing well inside its influence radius.

The double/multiple nuclei considered above have galactocentric separations that are below $r_{\text {infl }}$ but well above the separation at which the binary can be considered a "hard" binary. We conclude that the equilibrium and stability of such double/multiple nuclei could be understood in terms of the long dynamical friction timescale within the influence radius of the host galaxy SMBH predicted by our models.

We conclude by noting that the stalled satellites predicted by our models are likely to be on highly eccentric orbits (see Section 2). The highly eccentric orbit of the satellite can also have interesting observational consequences. As the satellite is disrupted, it will form an eccentric disk-like structure around the nucleus of the primary galaxy. A characteristic example could be the nucleus of the low-luminosity elliptical galaxy NGC 4486B (companion to M87). NGC 4486B is unusual for a galaxy of its luminosity in having a well-resolved core. Two brightness peaks are observed in the core separated by $\approx 12 \mathrm{pc}$ (Lauer et al. 1996). Neither peak is coincident with the galaxy photocenter, which falls between them. This double-peak structure has been interpreted as evidence for an eccentric disk of stars where the peaks would be the ansae of the disk orbiting an SMBH. The disk might be related to the disruption of a star cluster on an eccentric orbit by the tidal field of the primary $\mathrm{SMBH}$; its eccentric nature would naturally result by the dynamical friction process in the flat density core. For example, taking $M_{\bullet}=2 \times 10^{8} M_{\odot}$ (Lauer et al. 1996), an inspiraling star cluster with total mass $m=10^{6} M_{\odot}$ will reach the center in $\approx 0.2-0.5 \mathrm{Gyr}$ starting from $r_{\text {infl }}$. If we assume a reasonable value for the cluster core radius of $\approx 2 \mathrm{pc}$ then the cluster will be disrupted by the SMBH tidal field when it reaches a separation of $\approx 10 \mathrm{pc}$ from the center. This distance appears to be consistent with the observed offsets of the two nuclear brightness peaks in NGC 4486B.

\section{Conclusions}

In this paper, we studied the orbital evolution of a massive binary which consists of a massive object moving near an SMBH of considerably larger mass and which sits at the center of a galaxy. The main physical mechanism that drives the evolution of the binary is dynamical friction.

The main results of this paper are summarized below:

(1) We study the orbital evolution of the massive body, treating dynamical friction as a perturbation to the classic two-body problem. Unlike previous treatments, we take into account the contribution to dynamical friction of stars moving faster than the massive body. Assuming that the density profile of field stars follows $\rho \propto r^{-\gamma}$, we find that the binary secular eccentricity always increases unless $\gamma \gtrsim 2$. Specifically, low mass-ratio binaries with a moderate initial eccentricity, $e_{0} \gtrsim 0.3$, attain $e \gtrsim 0.9$ by the time they reach the hardening phase. Although the contribution from fast stars increases the orbital decay rate, for cusps shallower than $\rho \propto r^{-1}$ the dynamical friction timescale becomes very long.

(2) We run $N$-body simulations with different resolutions and for various slopes of the stellar cusp density profile and mass of the secondary body. We confirm the expected theoretical prediction that for shallow density profile cusps the eccentricity increases while for $\gamma \gtrsim 2$ the eccentricity decreases during the inspiral.

(3) We apply our treatment of dynamical friction to study the evolution of SMBH binaries formed in early-type galaxies. We treat independently the different phases involved in the evolution of the binary, compute the decay timescale that describes the dynamical friction phase, and calculate the lifetime of an SMBH binary as a 


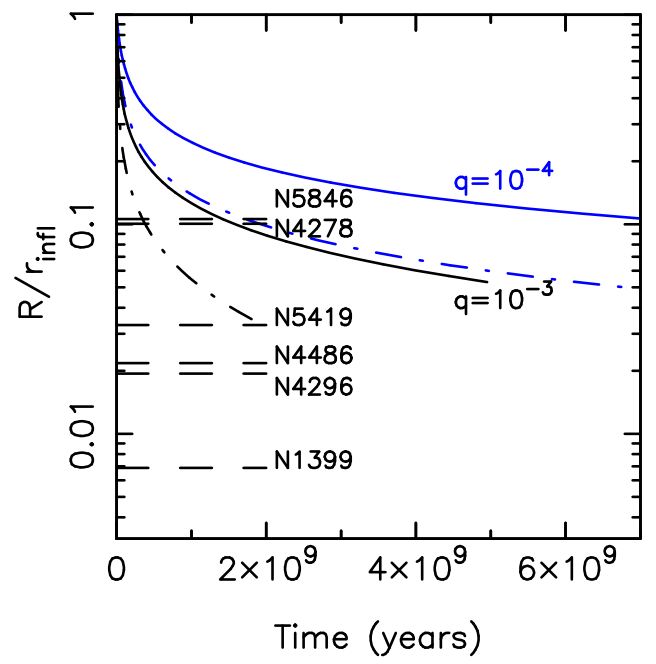

Figure 13. Evolution of orbital radius for $\gamma=0.6$ (solid lines) and $\gamma=1$ (dotted-dashed lines) and a binary of mass ratio $q=10^{-4}$ (black lines) and $q=10^{-3}$ (blue lines). Dashed horizontal lines give the value of the observed off-center displacement reported by Lena et al. (2014). The satellite galaxies stall at $\approx 0.1 r_{\text {infl. }}$. Stalled binaries could produce "displacements" comparable to those observed in NGC 4278 and NGC 5846, if the secondary SMBH is accreting. Note that the time has been normalized such that $M_{\bullet}=5 \times 10^{9} M_{\odot}$ and $r_{\text {infl }}=500 \mathrm{pc}$, but it can be rescaled to any of the galaxies we considered using $t \rightarrow t \times\left(r_{\text {infl }} / 500 \mathrm{pc}\right)^{3 / 2}\left(M_{\bullet} / 5 \times 10^{9} M_{\odot}\right)^{-1 / 2}$.

function of its total mass and mass ratio. We find that low mass-ratio binaries, $q \lesssim 10^{-3}$, formed in massive elliptical galaxies $(\gamma<1)$ have a lifetime greater than a Hubble time. This results in stalled satellite SMBHs on eccentric orbits at a galactocentric distance of order onetenth the influence radius of the primary black hole.

(4) We calculate the expected number of stalled satellites as a function of the host galaxy SMBH mass. We find that the number increases with the galaxy mass and that the brightest cluster galaxies should have a few of such satellites. We discuss our results in connection to displaced AGN, double and multiple nuclei often observed in core elliptical galaxies and eccentric nuclear stellar disks.

We want to thank our colleague Eugene Vasiliev, who provided insight and expertise that greatly assisted the research. We are thankful to the referee for comments and suggestions that helped to improve the manuscript during its revision stages.

\section{References}

Alessandrini, E., Lanzoni, B., Miocchi, P., Ciotti, L., \& Ferraro, F. R. 2014, ApJ, 795, 169

Alexander, T. 2005, PhR, 419, 65

Antonini, F. 2013, ApJ, 763, 62

Antonini, F., \& Merritt, D. 2012, ApJ, 745, 83

Arnalte Mur, P., Ellis, S. C., \& Colless, M. 2006, PASA, 23, 33

Baes, M., Buyle, P., Hau, G. K. T., \& Dejonghe, H. 2003, MNRAS, 341, L44 Batcheldor, D., Robinson, A., Axon, D. J., Perlman, E. S., \& Merritt, D. 2010, ApJL, 717, L6

Baumgardt, H., Gualandris, A., \& Portegies Zwart, S. 2006, MNRAS, 372, 174 Bédorf, J., Gaburov, E., \& Portegies Zwart, S. 2015, ComAC, 2, 8

Binney, J., \& Tremaine, S. 1987, Galactic Dynamics (Princeton, NJ: Princeton Univ. Press), 747

Bonfini, P., \& Graham, A. W. 2016, ApJ, 829, 81

Bundy, K., Fukugita, M., Ellis, R. S., et al. 2009, ApJ, 697, 1369

Burke-Spolaor, S. 2011, MNRAS, 410, 2113
Chandrasekhar, S. 1943, ApJ, 97, 255

Danby, J. J. A., \& Camm, G. L. 1957, MNRAS, 117, 50

de Ravel, L., Le Fèvre, O., Tresse, L., et al. 2009, A\&A, 498, 379

Dosopoulou, F., \& Kalogera, V. 2016a, ApJ, 825, 70

Dosopoulou, F., \& Kalogera, V. 2016b, ApJ, 825, 71

Dullo, B. T., \& Graham, A. W. 2012, ApJ, 755, 163

Dullo, B. T., \& Graham, A. W. 2015, ApJ, 798, 55

Farage, C. L., McGregor, P. J., Dopita, M. A., \& Bicknell, G. V. 2010, ApJ, 724, 267

Ferrarese, Laura, \& Merritt, David 2000, ApJL, 539, L9

Forbes, D. A., Lasky, P., Graham, A. W., \& Spitler, L. 2008, MNRAS, 389, 1924

Gaburov, E., Harfst, S., \& Portegies Zwart, S. 2009, NewA, 14, 630

Gebhardt, K., Bender, R., Bower, G., et al. 2000, ApJL, 539, L13

Gualandris, A., \& Merritt, D. 2008, ApJ, 678, 780

Haehnelt, M. G., \& Kauffmann, G. 2002, MNRAS, 336, L61

Harfst, S., Gualandris, A., Merritt, D., et al. 2007, NewA, 12, 357

Janowiecki, S., Mihos, J. C., Harding, P., et al. 2010, ApJ, 715, 972

Just, A., Khan, F. M., Berczik, P., Ernst, A., \& Spurzem, R. 2011, MNRAS, 411,653

Kalnajs, A. J. 1972, in IAU Coll. 10, Gravitational N-body Problems, ed. M. Lecar (Dordrecht: Reidel), 13

Kelley, L. Z., Blecha, L., \& Hernquist, L. 2016, arXiv:1606.01900

Khan, F. M., Just, A., \& Merritt, D. 2011, ApJ, 732, 89

Kitzbichler, M. G., \& White, S. D. M. 2008, MNRAS, 391, 1489

Komatsu, E., Smith, K. M., Dunkley, J., et al. 2011, ApJS, 192, 18

Kormendy, J., \& Ho, L. C. 2013, ARA\&A, 51, 511

Laine, S., van der Marel, R. P., Lauer, T. R., et al. 2003, AJ, 125, 478

Lauer, T. R., Ajhar, E. A., Byun, Y.-I., et al. 1995, AJ, 110, 2622

Lauer, T. R., Gebhardt, K., Richstone, D., et al. 2002, AJ, 124, 1975

Lauer, T. R., Tremaine, S., Ajhar, E. A., et al. 1996, ApJL, 471, L79

Lena, D., Robinson, A., Marconi, A., et al. 2014, ApJ, 795, 146

López-Sanjuan, C., Le Fèvre, O., Ilbert, O., et al. 2012, A\&A, 548, A7

Marconi, A., \& Hunt, L. K. 2003, ApJL, 589, L21

Matsubayashi, T., Makino, J., \& Ebisuzaki, T. 2007, ApJ, 656, 879

Mazzalay, X., Thomas, J., Saglia, R. P., et al. 2016, MNRAS, 462, 2847

McWilliams, S. T., Ostriker, J. P., \& Pretorius, F. 2014, ApJ, 789, 156

Merritt, D. 2006, ApJ, 648, 976

Merritt, D. 2013, Dynamics and Evolution of Galactic Nuclei (Princeton, NJ Princeton Univ. Press)

Merritt, D., \& Ferrarese, L. 2001, MNRAS, 320, L30

Merritt, D., Schnittman, J. D., \& Komossa, S. 2009, ApJ, 699, 1690

Mikkola, S., \& Valtonen, M. J. 1992, MNRAS, 259, 115

Mulder, W. A. 1983, A\&A, 117, 9

Nipoti, C., Treu, T., Leauthaud, A., et al. 2012, MNRAS, 422, 1714

Ostriker, E. C. 1999, ApJ, 513, 252

Paczynski, B. 1976, in IAU Symp. 73, Structure and Evolution of Close Binary Stems, ed. P. Eggleton, S. Mitton, \& J. Whelan (Dordrecht: Reidel), 75

Patton, D. R., \& Atfield, J. E. 2008, ApJ, 685, 235

Petts, J. A., Read, J. I., \& Gualandris, A. 2016, MNRAS, 463, 858

Postman, M., Lauer, T. R., Donahue, M., et al. 2012, ApJ, 756, 159

Rasskazov, A., \& Merritt, D. 2016, arXiv:1606.07484

Read, J. I., Goerdt, T., Moore, B., et al. 2006, MNRAS, 373, 1451

Rodriguez, C., Taylor, G. B., Zavala, R. T., et al. 2006, ApJ, 646, 49

Rodriguez, C., Taylor, G. B., Zavala, R. T., Pihlström, Y. M., \& Peck, A. B. 2009, ApJ, 697, 37

Rodriguez-Gomez, V., Genel, S., Vogelsberger, M., et al. 2015, MNRAS, 449,49

Sesana, A. 2013, MNRAS, 433, L1

Sparks, W. B., Macchetto, F., \& Golombek, D. 1989, ApJ, 345, 153

Spinnato, P. F., Fellhauer, M., \& Portegies Zwart, S. F. 2003, MNRAS, 344, 22

Tremaine, S., \& Weinberg, M. D. 1984, MNRAS, 209, 729

Valtaoja, E., Teräsranta, H., Tornikoski, M., et al. 2000, ApJ, 531, 744

van den Bosch, F. C., Lewis, G. F., Lake, G., \& Stadel, J. 1999, ApJ, 515, 50

Vasiliev, E. 2015, MNRAS, 446, 3150

Vasiliev, E., Antonini, F., \& Merritt, D. 2014, ApJ, 785, 163

Vasiliev, E., Antonini, F., \& Merritt, D. 2015, ApJ, 810, 49

Weinberg, M. D. 1986, ApJ, 300, 93

Xu, C. K., Zhao, Y., Scoville, N., et al. 2012, ApJ, 747, 85

Yu, Q. 2002, MNRAS, 331, 935 